Pacific

Journal of

Mathematics

\title{
LEFSCHETZ PENCILS AND FINITELY PRESENTED GROUPS
}

Ryoma Kobayashi AND NAOYUKi Monden 


\title{
LEFSCHETZ PENCILS AND FINITELY PRESENTED GROUPS
}

\author{
RYOMA KobAYASHI AND NAOYUKi MONDEN
}

\begin{abstract}
From the works of Gompf and Donaldson, it is known that every finitely presented group can be realized as the fundamental group of the total space of a Lefschetz pencil. We give an alternative proof of this fact by providing the monodromy explicitly. In the proof, we give an alternative construction of the monodromy of Gurtas' fibration and a lift of that to the mapping class group of a surface with two boundary components.
\end{abstract}

\section{Introduction}

There exist Lefschetz pencils (fibrations over $S^{2}$ with (-1)-sections) whose total spaces have a prescribed fundamental group. This follows as a corollary of the results of Gompf [1995], who showed that every finitely presented group is realized as the fundamental group of some closed symplectic 4-manifold, and of Donaldson [1999], who showed that every closed symplectic 4-manifold admits a Lefschetz pencil. Note that since we obtain a Lefschetz fibration with $(-1)$-sections by blowing up the base locus of a Lefschetz pencil, and blowing up has no effect on the fundamental groups of 4-manifolds, the above claim for Lefschetz fibrations with $(-1)$-sections follows. Conversely, a 4-manifold admitting a Lefschetz pencil (fibration with fiber genus greater than one) is symplectic (cf. [Gompf and Stipsicz 1999]).

Let $\Sigma_{g}^{b}$ be a compact oriented surface of genus $g$ with $b$ boundary components $\delta_{1}, \ldots, \delta_{b}$, and let $\operatorname{Mod}_{g}^{b}$ be the mapping class group of $\Sigma_{g}^{b}$. We denote by $t_{c}$ the right-handed Dehn twist along a simple closed curve $c$ in $\Sigma_{g}^{b}$. Then a relation $\prod_{j=1}^{b} t_{\delta_{j}}=\prod_{i=1}^{m} t_{v_{i}}$ provides a genus- $g$ Lefschetz pencil/fibration with $b$ base points/(-1)-sections. Conversely, given any Lefschetz pencil (fibration with (-1)sections), we obtain such a relation. However, the relations corresponding to the above Lefschetz pencils/fibrations constructed based on the results of [Gompf 1995] and [Donaldson 1999] are implicit. Our purpose is to provide the relation of such a genus- $g$ Lefschetz pencil explicitly, so this gives an alternative proof of the above corollary using mapping class group arguments. To state our main result, we need to introduce some notation.

MSC2010: primary 57R17; secondary 20F34.

Keywords: Lefschetz pencil, Lefschetz fibration, fundamental group, mapping class group. 
Definition 1.1. Let $\Gamma=\left\langle x_{1}, x_{2}, \ldots, x_{n} \mid r_{1}, r_{2}, \ldots, r_{k}\right\rangle$ be a finitely presented group with $n$ generators and $k$ relations. For $w \in \Gamma$, we define $l(w)$, called the syllable length of $w$, to be

$$
l(w)=\min \left\{s \mid w=x_{i_{1}}^{m_{1}} x_{i_{2}}^{m_{2}} \cdots x_{i_{s}}^{m_{s}} \text { for } 1 \leq i_{j} \leq n \text { and } m_{j} \in \mathbb{Z}\right\} .
$$

Define $l=\max \left\{l\left(r_{i}\right) \mid 1 \leq i \leq k\right\}$. If $k=0$, we define $l=1$ (note that $l$ depends on the presentation and that our definition of $l$ differs from that of [Korkmaz 2009]). We always assume that the relators $r_{i}$ are cyclically reduced.

In Section 5A, we give a relation $t_{\delta_{1}} t_{\delta_{2}}=W_{2}^{g}\left(1, \psi_{k}\right)$ in $\operatorname{Mod}_{g}^{2}$ using certain substitution techniques, where $W_{2}^{g}\left(1, \psi_{k}\right)$ is a product of right-handed Dehn twists. Our main result is the following:

Theorem 1.2. If $k \geq 1$ (resp. $k=0)$, then, for $g \geq 4(n+l-1)+k$ (resp. $g \geq$ $4 n+2)$, there exists a genus- $g$ Lefschetz pencil/fibration with two base points/(-1)sections on a closed symplectic 4-manifold X such that $t_{\delta_{1}} t_{\delta_{2}}=W_{2}^{g}\left(1, \psi_{k}\right)$ is the corresponding relation and $\pi_{1}(X)$ is isomorphic to $\Gamma$.

Theorem 1.2 gives an upper bound for the minimum $g$, denoted by $g_{P}(\Gamma)$, for which there exists a genus- $g$ Lefschetz pencil on $X$ such that $\pi_{1}(X)$ is isomorphic to $\Gamma$. We describe it in Section 8. To give a better upper bound on $g_{P}(\Gamma)$, we construct a lift of Gurtas' positive relator (see [Gurtas 2004]), denoted by $\theta^{2}$, to $\operatorname{Mod}_{g}^{2}$ in Section 6 by combining a lift of a hyperelliptic involution and the relation given in [Korkmaz 2009] to $\operatorname{Mod}_{g}^{2}$. On the other hand, Gurtas showed that the positive word $\theta^{2}$ given in [Gurtas 2004] is a positive relator by checking the images of certain cycles on $\Sigma_{g}$ under $\theta$. In this sense, our construction of the monodromy of Gurtas' fibration is different from that in [Gurtas 2004].

Here, we explain why we focus on Lefschetz fibrations with (-1)-sections. A section of a Lefschetz fibration over $S^{2}$ plays important roles in the total space. The existence of a section $\sigma$ of a Lefschetz fibration $f: X \rightarrow S^{2}$ with a fiber $F$ is required to compute the fundamental group of $X$ and to decide whether $X$ is spin or not (see [Gompf and Stipsicz 1999; Stipsicz 2001b]). In addition, the complement of a regular neighborhood of $F \cup \sigma$ is a Stein filling of its boundary equipped with the induced tight contact structure (see [Akbulut and Ozbagci 2002; Etnyre and Honda 2002; Loi and Piergallini 2001]). Especially, a (-1)-section is important in Lefschetz fibrations in the following senses.

(i) Blowing up of the base locus of a Lefschetz pencil yields a Lefschetz fibration with $(-1)$-sections. Conversely, we can obtain a Lefschetz pencil by blowing down of $(-1)$-sections of a Lefschetz fibration.

(ii) From given Lefschetz fibrations, we can construct a new Lefschetz fibration by fiber summing them. If a Lefschetz fibration admits a (-1)-section, it cannot be decomposed as any nontrivial fiber sum (see [Stipsicz 2001a; Smith 2001]). 
For these reasons, we can regard Lefschetz fibrations with (-1)-sections as "fundamental" and "prime" ones.

Note that we can express Gompf's result in terms of Lefschetz fibrations over $S^{2}$. The article [Amorós et al. 2000] gave a construction of Lefschetz fibrations whose total spaces have a given fundamental group without using Donaldson's result. However, their monodromies are implicit. The explicit monodromies of such fibrations were given by Korkmaz [2009]. Akhmedov and Ozbagci [2013] gave a new construction of such fibrations, and the first author [Kobayashi 2015] improved the result of [Korkmaz 2009]. For technical reasons, the fibrations in [Korkmaz 2009; Akhmedov and Ozbagci 2013; Kobayashi 2015] have no (-1)-sections (see Section 8), so we would like to emphasize that our result is different from the above four results.

Here is an outline of this paper. In Section 2, we fix notation. In Section 3, we introduce a substitution technique and the relation constructed by Korkmaz. Section 4 reviews some standard facts on Lefschetz fibrations and pencils. In Section 5, we prove the main results. In Section 6, we give an alternative construction of the monodromy of Gurtas' fibration and provide a lift of that to the mapping class group of a surface with two boundary components. In Section 7, we introduce the construction of a loop which is needed for the proof of Theorem 1.2. In Section 8, we give an upper bound of $g_{P}(\Gamma)$ and some remarks.

\section{Notation}

Let $\Sigma_{g}$ be the closed oriented surface of genus $g$ standardly embedded in 3-space as shown in Figure 1. We use the symbols $a_{1}, b_{1}, \ldots, a_{g}, b_{g}$ to denote the standard generators of the fundamental group $\pi_{1}\left(\Sigma_{g}\right)$ of $\Sigma_{g}$. For $a$ and $b$ in $\pi_{1}\left(\Sigma_{g}\right)$, the notation $a b$ means that we first apply $a$ then $b$.

Let $c_{0}, c_{1}, c_{2}, \ldots, c_{g}, a_{0}, a_{g+1}, a_{0}^{\prime}, a_{g+1}^{\prime}$ be the simple loops in $\Sigma_{g}$ depicted in Figure 1. Note that in $\pi_{1}\left(\Sigma_{g}\right)$, up to conjugation,

$$
c_{i}=b_{i}^{-1} \cdots b_{1}^{-1}\left(a_{1} b_{1} a_{1}^{-1}\right) \cdots\left(a_{i} b_{i} a_{i}^{-1}\right) \quad \text { for each } 1 \leq i \leq g \text {; }
$$

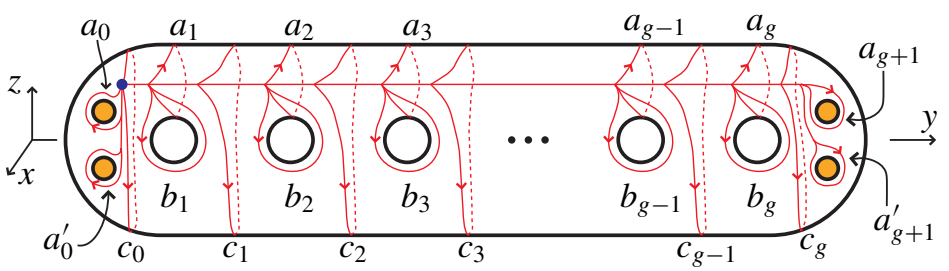

Figure 1. Generators $a_{j}, b_{j}$ of the fundamental group and loops $c_{j}, a_{0}^{\prime}, a_{g+1}^{\prime}$. 
as well as

$$
\begin{aligned}
& c_{0}=c_{g}=1, \\
& a_{0}=a_{g+1}=a_{0}^{\prime}=a_{g+1}^{\prime}=1 .
\end{aligned}
$$

Then the fundamental group $\pi_{1}\left(\Sigma_{g}\right)$ has the presentation

$$
\pi_{1}\left(\Sigma_{g}\right)=\left\langle a_{1}, b_{1}, \ldots, a_{g}, b_{g} \mid c_{g}\right\rangle .
$$

Let $B_{0}, B_{1}, B_{2}, \ldots, B_{g}, a_{1}^{\prime}, \ldots, a_{g}^{\prime}$ be the simple closed curves in $\Sigma_{g}$ shown in Figure 2. Suppose that $g=2 r$. Then it is easy to check that, up to conjugation, the following equalities hold in $\pi_{1}\left(\Sigma_{g}\right)$ :

$$
\begin{aligned}
B_{2 k-1} & =a_{k} b_{k} b_{k+1} \cdots b_{g+1-k} c_{g+1-k} a_{g+1-k} & & \text { for } 1 \leq k \leq r \\
B_{2 k} & =a_{k} b_{k+1} b_{k+2} \cdots b_{g-k} c_{g-k} a_{g+1-k} & & \text { for } 0 \leq k \leq r \\
a_{k+1}^{\prime} & =c_{k} a_{k+1} & & \text { for } 0 \leq k \leq g-1 .
\end{aligned}
$$

If $g=2 r+1$, then $B_{2 k-1}$ satisfies the equality (4) for $1 \leq k \leq r+1$.

Let $A_{1}, \ldots, A_{2 g+1}$ be the simple closed curves on $\Sigma_{g}$ shown in Figure 3 . It is easily seen that, up to conjugation, the following equalities hold in $\pi_{1}\left(\Sigma_{g}\right)$ :

$$
\begin{aligned}
& A_{2 k}=b_{k} \quad \text { for } 1 \leq k \leq g ； \\
& A_{2 k+1}=a_{k} a_{k+1}^{-1} \quad \text { for } 0 \leq k \leq g \text {. }
\end{aligned}
$$

Moreover, when we denote by $D_{0}, D_{1}, D_{2}, \ldots, D_{2 h_{1}}$ and $E_{h_{1}}$ the simple closed curves on $\Sigma_{g}$ indicated in Figure 3, it is immediate that, up to conjugation, the
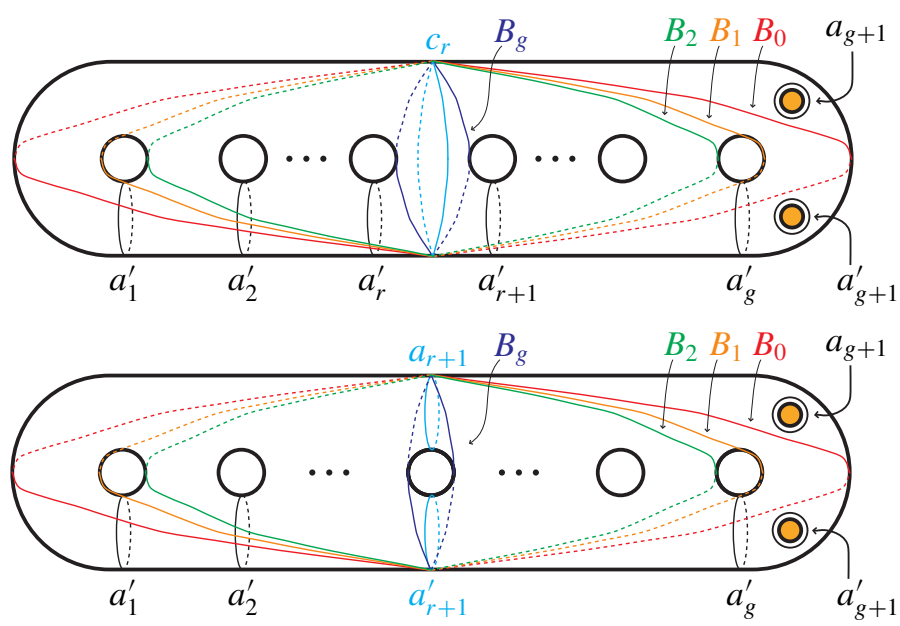

Figure 2. The curves $B_{0}, B_{1}, B_{2}, \ldots, B_{g}, a_{1}^{\prime}, \ldots, a_{g}^{\prime}$. 


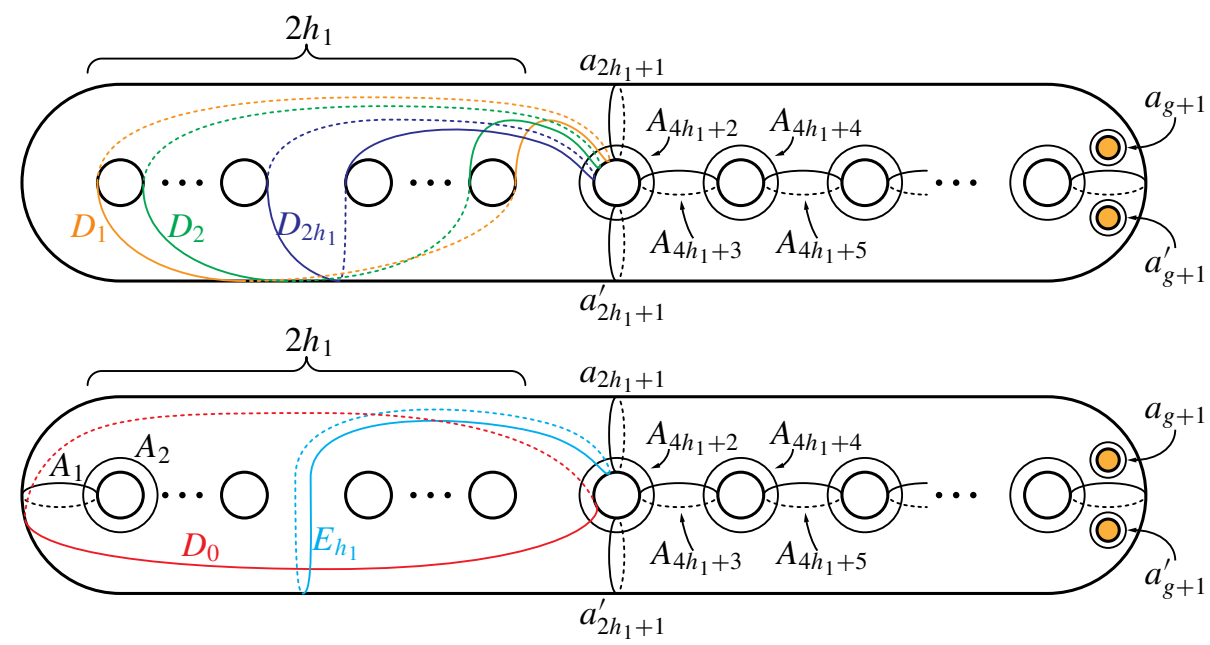

Figure 3. The curves $A_{1}, A_{2}, \ldots, A_{2 g+1}, D_{0}, D_{1}, \ldots, D_{2 h_{1}}$ and $E_{h_{1}}$.

following equalities hold in $\pi_{1}\left(\Sigma_{g}\right)$ :

$$
\begin{array}{rlrl}
D_{0} & =b_{1} b_{2} \cdots b_{2 h_{1}} a_{2 h_{1}+1}^{-1} ; & \\
D_{2 k-1} & =a_{k} b_{k} b_{k+1} \cdots b_{2 h_{1}+1-k} c_{2 h_{1}+1-k} a_{2 h_{1}+1-k} a_{2 h_{1}+1}^{-1} & & \text { for } 1 \leq k \leq h_{1} ; \\
D_{2 k} & =a_{k} b_{k+1} b_{k+2} \cdots b_{2 h_{1}-k} c_{2 h_{1}-k} a_{2 h_{1}+1-k} a_{2 h_{1}+1}^{-1} & & \text { for } 1 \leq k \leq h_{1} ; \\
E_{h_{1}} & =c_{h_{1}} a_{2 h_{1}+1} . & &
\end{array}
$$

Note that we can modify $\Sigma_{g}$ and $D_{0}, D_{1}, D_{2}, \ldots, D_{2 h_{1}}, E_{h_{1}}$ by isotopy as in Figure 4.

Throughout this paper, we use the same symbol for a loop and its homotopy class. Similarly, we use the same symbol for a diffeomorphism and its isotopy class, or a simple closed curve and its isotopy class. A simple loop and a simple closed curve will even be denoted by the same symbol. It will cause no confusion as it will be clear from the context which one we mean.

\section{Mapping class groups}

3A. Substitution techniques. Let $\Sigma_{g}^{b}$ be a compact oriented surface of genus $g$ with $b$ boundary components. The mapping class group of $\Sigma_{g}^{b}$, which we denote by $\operatorname{Mod}_{g}^{b}$, is the group of isotopy classes of orientation preserving selfdiffeomorphisms of $\Sigma_{g}^{b}$. We assume that diffeomorphisms and isotopies fix the points of the boundary. To simplify notation, we write $\Sigma_{g}=\Sigma_{g}^{0}$ and $\operatorname{Mod}_{g}=\operatorname{Mod}_{g}^{0}$. For $\phi_{1}$ and $\phi_{2}$ in $\operatorname{Mod}_{g}^{b}$, the notation $\phi_{1} \phi_{2}$ means that we first apply $\phi_{2}$ then $\phi_{1}$ (Our notation differs from that of [Korkmaz 2009].) Let $t_{c}$ be the Dehn twist about 


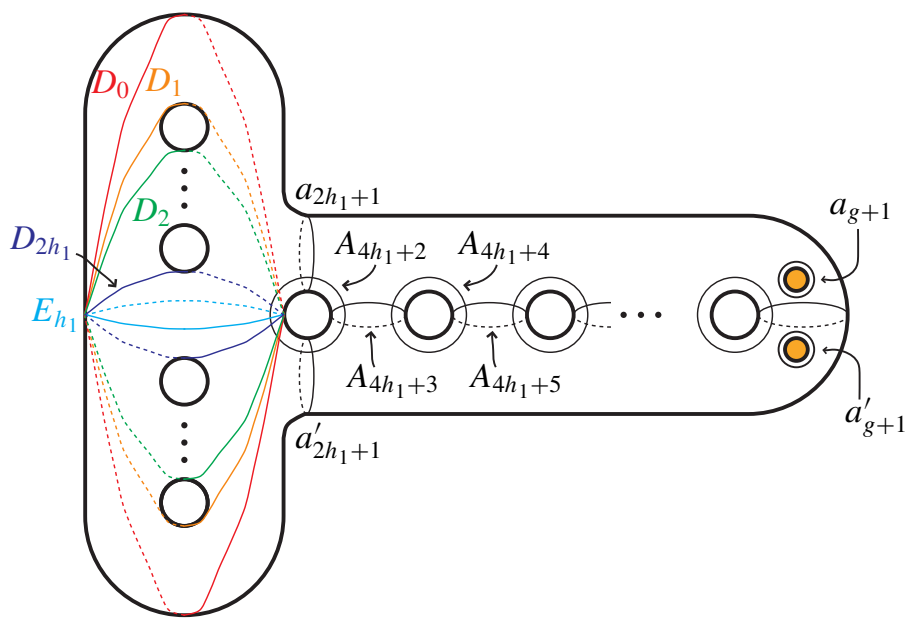

Figure 4. Modified surface $\Sigma_{g}$ and modified curves $D_{0}, D_{1}, \ldots, D_{2 h_{1}}$ and $E_{h_{1}}$.

a simple closed curve $c$ in $\Sigma_{g}^{b}$. Note that $t_{\phi(c)}=\phi t_{c} \phi^{-1}$ for an element $\phi$ in $\operatorname{Mod}_{g}^{b}$ and $t_{c} t_{d}=t_{d} t_{c}$ if $c$ is disjoint from $d$.

Definition 3.1. A word $\varrho:=t_{c_{1}} t_{c_{2}} \cdots t_{c_{n}}$ in $\operatorname{Mod}_{g}$ is called a positive relator if $\varrho$ satisfies $\varrho=1$.

We introduce a primary technique to construct new products of right-handed Dehn twists in $\operatorname{Mod}_{g}^{b}$ from old ones.

Definition 3.2. Let $\phi$ be an element in $\operatorname{Mod}_{g}^{b}$. Write

$$
W=t_{c_{1}} t_{c_{2}} \cdots t_{c_{k}}, \quad W^{\phi}=t_{\phi\left(c_{1}\right)} t_{\phi\left(c_{2}\right)} \cdots t_{\phi\left(c_{k}\right)}, \quad V=t_{d_{1}} t_{d_{2}} \cdots t_{d_{l}} .
$$

If the relation $V=W$ holds in $\operatorname{Mod}_{g}^{b}$ and $\phi\left(d_{i}\right)=d_{i}$ for all $i$, then by $t_{\phi(c)}=\phi t_{c} \phi^{-1}$ we obtain the relation

$$
V=W^{\phi} .
$$

in $\operatorname{Mod}_{g}^{b}$. Let $\varrho$ be a product of right-handed Dehn twists which includes $V$ as a subword:

$$
\varrho:=U_{1} \cdot V \cdot U_{2},
$$

where $U_{1}$ and $U_{2}$ are products of right-handed Dehn twists. Then we get a new product $\varsigma(\phi)$ of right-handed Dehn twists

$$
\varsigma(\phi):=U_{1} \cdot W^{\phi} \cdot U_{2},
$$

and $\varsigma(\phi)$ is said to be obtained by applying a $W^{\phi}$-substitution of $V$ to $\varrho$. 
Remark 3.3. Fuller introduced the above operation for $\phi=$ id. Auroux [2006b; 2006a] introduced the operation to obtain $\varsigma(\phi)$ from $\varsigma$ (id), called a "partial conjugation" by $\phi$. In a previous paper, we call the operation in Definition 3.2 a "twisted substitution”. As B. Ozbagci and R. I. Baykur kindly pointed out to us, the twisted substitution is a combination of these two operations.

3B. The word $W_{2}^{g}$. In this section, we introduce a word $W_{2}^{g}$ in $\operatorname{Mod}_{g}^{2}$. We denote by $\Sigma_{g}^{2}$ the surface of genus $g$ with two boundary components obtained from $\Sigma_{g}$ by removing two disjoint open disks bounded by $a_{g+1}$ and $a_{g+1}^{\prime}$ (cf. Figure 1 and 2), so $a_{g+1}$ and $a_{g+1}^{\prime}$ are the boundary curves of $\Sigma_{g}^{2}$. Set

$$
W_{2}^{g}:= \begin{cases}\left(t_{B_{0}} t_{B_{1}} t_{B_{2}} \cdots t_{B_{g}} t_{c_{r}}\right)^{2} & \text { if } g=2 r, \\ \left(t_{B_{0}} t_{B_{1}} t_{B_{2}} \cdots t_{B_{g}} t_{a_{r+1}}^{2} t_{a_{r+1}^{\prime}}^{2}\right)^{2} & \text { if } g=2 r+1 .\end{cases}
$$

Korkmaz [2009] gave the following relation:

Lemma 3.4 [Korkmaz 2009]. We have $t_{a_{g+1}} t_{a_{g+1}^{\prime}}=W_{2}^{g}$ in $\operatorname{Mod}_{g}^{2}$.

Although Korkmaz does not prove Lemma 3.4, we can prove it by applying the same argument as in Section 2 of [Korkmaz 2001]. In Section 6A, we give a very short outline of the proof. Since the simple closed curves $a_{g+1}$ and $a_{g+1}^{\prime}$ are null-homotopic in $\Sigma_{g}$, it follows that $t_{a_{g+1}}=t_{a_{g+1}^{\prime}}=1$ in $\operatorname{Mod}_{g}$. Therefore, the word $W_{2}^{g}$ in $\operatorname{Mod}_{g}$ is a positive relator. This positive relator for $g=2$ was discovered by Matsumoto [1996], and its generalization was constructed independently by Cadavid [1998] and Korkmaz [2001].

\section{Lefschetz pencils and fibrations}

We recall the definition and basic properties of Lefschetz pencils and fibrations. More details can be found in [Gompf and Stipsicz 1999].

Definition 4.1. Let $X$ be a closed, connected, oriented smooth 4-manifold, and let $B=\left\{b_{1}, \ldots, b_{m}\right\}$ and $C=\left\{p_{1}, \ldots, p_{n}\right\}$ be finite, disjoint subsets of $X$.

Let $f: X \backslash B \rightarrow S^{2}$ be a smooth map satisfying the following three conditions:

(a) For each $b_{i} \in B$, called the base point, there are orientation-preserving complex coordinate charts on which $f$ is of the form $f\left(z_{1}, z_{2}\right)=z_{1} / z_{2}$.

(b) $C$ is the set of critical points of $f$, and for each $p_{i}$ and $f\left(p_{i}\right)$, there are complex local coordinate charts agreeing with the orientations of $X$ and $S^{2}$ on which $f$ is of the form $f\left(z_{1}, z_{2}\right)=z_{1} z_{2}$.

(c) For $q \in S^{2}-f(C)$, the set $f^{-1}(q) \cup B \subset X$ is diffeomorphic to $\Sigma_{g}$.

Then $f$ is called a genus- $g$ Lefschetz pencil if $B$ is a nonempty set, and $f$ is called a genus- $g$ Lefschetz fibration if $B$ is the empty set. 
The set $B$ is called the base locus, and for each $q \in S^{2}$, the set $f(q)^{-1} \cup B$ is called the fiber of $f$. We assume that $f$ is injective on $C$ and that $f$ is relatively minimal (i.e., no fiber contains a sphere with self-intersection number -1). A fiber containing a critical point is called a singular fiber. Each singular fiber is obtained by collapsing a simple closed curve, called the vanishing cycle, in the regular fiber to a point.

Once we fix an identification of $\Sigma_{g}$ with the fiber over a base point of $S^{2}-f(C)$, we can characterize the Lefschetz fibration $f: X \rightarrow S^{2}$ by its monodromy representation $\pi_{1}\left(S^{2}-f(C)\right) \rightarrow \operatorname{Mod}_{g}$. Note that in this paper, this map is an antihomomorphism. Let $\gamma_{1}, \ldots, \gamma_{n}$ be an ordered system of generating loops for $\pi_{1}\left(S^{2}-f(C)\right)$, such that each $\gamma_{i}$ encircles only $f\left(p_{i}\right)$ and $\gamma_{1} \gamma_{2} \cdots \gamma_{n}$ is homotopically trivial. Thus, since the monodromy of the fibration along each of the loops $\gamma_{i}$ is a right-handed Dehn twist along the corresponding vanishing cycle, the monodromy of $f$ comprises a positive relator

$$
t_{v_{n}} \cdots t_{v_{2}} t_{v_{1}}=1 \in \operatorname{Mod}_{g},
$$

where the $v_{i}$ are the corresponding vanishing cycles of the singular fibers. Conversely, for any positive relator $\varrho \in \operatorname{Mod}_{g}$, we can construct a genus- $g$ Lefschetz fibration over $S^{2}$ whose monodromy is $\varrho$. Therefore, we denote a genus- $g$ Lefschetz fibration associated to a positive relator $\varrho$ in $\operatorname{Mod}_{g}$ by $f_{\varrho}: X_{\varrho} \rightarrow S^{2}$.

Definition 4.2. For a Lefschetz fibration $f: X \rightarrow S^{2}$, a map $\sigma: S^{2} \rightarrow X$ is called a $k$-section of $f$ if $f \circ \sigma=\mathrm{id}_{S^{2}}$ and the self-intersection number of the homology class $\left[\sigma\left(S^{2}\right)\right]$ in $H_{2}(X ; \mathbb{Z})$ is equal to $k$.

When a Lefschetz fibration $X \rightarrow S^{2}$ admits a section, we can compute the fundamental group of $X$ as follows.

Lemma 4.3 (cf. [Gompf and Stipsicz 1999]). Let @ be a positive relator given by $t_{v_{n}} \cdots t_{v_{2}} t_{v_{1}}=1$ in $\operatorname{Mod}_{g}$. Suppose that a genus-g Lefschetz fibration $f_{\varrho}: X_{\varrho} \rightarrow S^{2}$ admits a section $\sigma$. Then the fundamental group $\pi_{1}\left(X_{\varrho}\right)$ is isomorphic to the quotient of $\pi_{1}\left(\Sigma_{g}\right)$ by the normal subgroup generated by $v_{1}, \ldots, v_{n}$.

From the definitions of Lefschetz fibrations and pencils, blowing up all points of $B=\left\{q_{1}, \ldots, q_{b}\right\}$ of a genus- $g$ Lefschetz pencil yields a genus- $g$ Lefschetz fibration with $b$ disjoint $(-1)$-sections. Let $\delta_{1}, \delta_{2}, \ldots, \delta_{b}$ be $b$ boundary curves of $\Sigma_{g}^{b}$. Then a lift of a positive relator $\varrho$ in $\operatorname{Mod}_{g}$, namely $t_{v_{n}} \cdots t_{v_{2}} t_{v_{1}}=1$, to $\operatorname{Mod}_{g}^{b}$ as

$$
t_{v_{n}^{\prime}} \cdots t_{v_{2}^{\prime}} t_{v_{1}^{\prime}}=t_{\delta_{1}} t_{\delta_{2}} \cdots t_{\delta_{b}}
$$

shows the existence of $b$ disjoint $(-1)$-sections of $f_{\varrho}$. Here, $v_{i}^{\prime}$ is a simple closed curve mapped to $v_{i}$ under $\Sigma_{g}^{b} \rightarrow \Sigma_{g}$. Conversely, such a relation determines a genus- $g$ Lefschetz fibration with $m$ disjoint $(-1)$-sections and a genus- $g$ Lefschetz pencil by blowing these sections down. 


\section{Proof of Theorem 1.2}

For a finitely presented group $\Gamma=\left\langle x_{1}, x_{2}, \ldots, x_{n} \mid r_{1}, r_{2}, \ldots, r_{k}\right\rangle$ with $n$ generators and $k$ relators, let $l=\max \left\{l\left(r_{i}\right) \mid 1 \leq i \leq k\right\}$, where $l\left(r_{i}\right)$ is the syllable length of $r_{i}$. In this section, we denote by $h_{1}$ and $h_{2}$ two integers satisfying $h_{1} \geq n+l-1$ and $2\left(h_{2}-1\right) \geq k$, respectively.

5A. Construction of a word $W_{2}^{g}\left(1, \psi_{i}\right)$. In this subsection, we construct a key relation in $\operatorname{Mod}_{g}^{2}$.

Let us consider $\Sigma_{g}^{2}$ obtained from $\Sigma_{g}$ by removing two disjoint open disks surrounded by $a_{g+1}$ and $a_{g+1}^{\prime}$ (see Section 2 and Figures 1-3). Write $r=2 h_{1}+h_{2}-1$ and $g=2 r$ or $2 r+1$. For $h_{2}-1 \geq 1$, we set

$$
\begin{aligned}
& X=t_{A_{4 h_{1}+2}} t_{A_{4 h_{1}+3}} \cdots t_{A_{2 r}}, \\
& \bar{X}=t_{A_{2 r}} \cdots t_{A_{4 h_{1}+3}} t_{A_{4 h_{1}+2}}, \\
& Y=\left(t_{D_{0}} t_{D_{1}} \cdots t_{D_{2 h_{1}}}\right)^{2} .
\end{aligned}
$$

Moreover, we define words $V_{1}$ and $V_{2}$ to be

$$
\begin{aligned}
& V_{1}=t_{E_{h_{1}}} X t_{a_{r}} t_{a_{r}^{\prime}} \bar{X} t_{E_{h_{1}}} t_{a_{r}^{\prime}} \bar{X} Y X t_{a_{r}^{\prime}}, \\
& V_{2}=t_{E_{h_{1}}} X t_{a_{r}} t_{a_{r}^{\prime}} \bar{X} t_{E_{h_{1}}} t_{A_{2 r+1}} \bar{X} Y X t_{A_{2 r+1}} .
\end{aligned}
$$

Then we obtain the relations in the following proposition.

Proposition 5.1. We have $t_{c_{r}}=V_{1}$ and $t_{a_{r+1}} t_{a_{r+1}^{\prime}}=V_{2}$ in $\operatorname{Mod}_{g}^{2}$.

We postpone the proof of Proposition 5.1 until Section 6 (see Proposition 6.1).

Let $h_{1} \geq n+l-1$ and $2\left(h_{2}-1\right) \geq k$. The next proposition is needed to prove Theorem 1.2.

Proposition 5.2. Let $F_{n}$ be the subgroup of $\pi_{1}\left(\Sigma_{g}\right)$ generated by the generators $a_{1}, \ldots, a_{n}$, i.e., $F_{n}$ is a free group of rank $n$. Let $r_{1}, \ldots, r_{k}$ be $k$ elements in $F_{n}$ represented as words in $a_{1}, \ldots, a_{n}$. Let $l=\max _{1 \leq i \leq k}\left\{l\left(r_{i}\right)\right\}$, where $l\left(r_{i}\right)$ is the syllable length of $r_{i}$. Then there are simple loops $R_{1}, \ldots, R_{k}$ in $\Sigma_{g}$ (see Figure 5) with the property that, for $4 h_{1}+2 \leq j \leq 4 h_{1}+2 h_{2}-2$ and $1 \leq i \leq k$,

(a) $R_{i}$ is disjoint from $A_{2 h_{1}+1}, \ldots, A_{4 h_{1}}, c_{2 h_{1}+h_{2}-1}\left(=c_{r}\right)$.

(b) $R_{1}$ intersects $a_{2 h_{1}+h_{2}-1}$ at one point and does not intersect $A_{j}$ for any $j$.

(c) $R_{i}$ intersects $A_{4 h_{1}+2 h_{2}-i}$ at one point and intersects neither $a_{2 h_{1}+h_{2}-1}$ nor $A_{j}$ for any $j \neq 4 h_{1}+2 h_{2}-i$ and $i \geq 2$.

(d) $\Phi\left(\left[R_{i}\right]\right)=r_{i}$, where $\left[R_{i}\right] \in \pi_{1}\left(\Sigma_{g}\right)$ is the homotopy class of the loop $R_{i}$, and $\Phi: \pi_{1}\left(\Sigma_{g}\right) \rightarrow \pi_{1}\left(\Sigma_{n}\right)$ is the map defined by $\Phi\left(a_{m}\right)=a_{m}$ for $1 \leq m \leq n$ and $\Phi(\alpha)=1$ for $\alpha \in\left\{a_{n+1}, \ldots, a_{g}, b_{1}, \ldots, b_{g}\right\}$. 


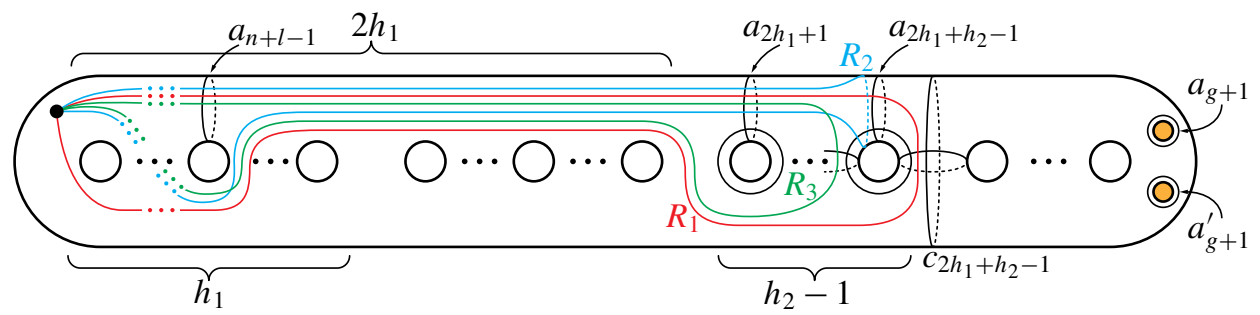

Figure 5. Curves $R_{1}, \ldots, R_{k}$ in $\Sigma_{g}$.

In Section 7, we prove Proposition 5.2 by constructing simple loops $R_{1}, \ldots, R_{k}$ explicitly. We also consider the loops $R_{1}, \ldots, R_{k}$ as simple loops on $\Sigma_{g}^{2}$ by removing two disjoint open disks surrounded by $a_{g+1}, a_{g+1}^{\prime}$ from $\Sigma_{g}$ (see Figure 5).

For $i=0,1, \ldots, k$, we define an element $\psi_{i}$ in $\operatorname{Mod}_{g}^{2}$ to be

$$
\begin{aligned}
& \psi_{0}=t_{a_{h_{1}}} t_{b_{h_{1}+1}} t_{b_{h_{1}+2}} \cdots t_{b_{2 h_{1}}}, \\
& \psi_{i}=t_{R_{k+1-i}} t_{R_{k+2-i}} \cdots t_{R_{k}} \psi_{0},
\end{aligned}
$$

where the $R_{i}$ are the loops on $\Sigma_{g}^{2}$ described above. From Proposition 5.2, for each $i$, we see that $\psi_{i}\left(c_{r}\right)=c_{r}$ if $g=2 r$, while $\psi_{1}\left(a_{r+1}\right)=a_{r+1}$ and $\psi_{1}\left(a_{r+1}^{\prime}\right)=a_{r+1}^{\prime}$ if $g=2 r+1$.

If $g=2 r$, then we can find two $t_{c_{r}}$ in the word $W_{2}^{g}$. By Proposition 5.1, we can apply $V_{1}^{\text {id }}$-substitution for one $t_{c_{r}}$ and $V_{1}^{\psi_{i}}$-substitution for the other.

If $g=2 r+1$, then since $t_{a_{r+1}}^{2} t_{a_{r+1}^{\prime}}^{2}=\left(t_{a_{r+1}} t_{a_{r+1}^{\prime}}\right)^{2}$, we can find four $t_{a_{r+1}} t_{a_{r+1}^{\prime}}$ in the word $W_{2}^{g}$. By Proposition 5.1, we can apply $V_{2}^{\text {id }}$-substitution for one $t_{a_{r+1}} t_{a_{r+1}^{\prime}}$ and $V_{2}^{\psi_{i}}$-substitution for the other.

If we set

$$
W_{2}^{g}\left(1, \psi_{i}\right):=\left(t_{B_{0}} t_{B_{1}} t_{B_{2}} \cdots t_{B_{g}} V_{1}\right)\left(t_{B_{0}} t_{B_{1}} t_{B_{2}} \cdots t_{B_{g}} V_{1}^{\psi_{i}}\right)
$$

if $g=2 r$, and

$$
W_{2}^{g}\left(1, \psi_{i}\right):=\left(t_{B_{0}} t_{B_{1}} t_{B_{2}} \cdots t_{B_{g}} t_{a_{r+1}} t_{a_{r+1}^{\prime}} V_{2}\right)\left(t_{B_{0}} t_{B_{1}} t_{B_{2}} \cdots t_{B_{g}} t_{a_{r+1}} t_{a_{r+1}^{\prime}} V_{2}^{\psi_{i}}\right)
$$

if $g=2 r+1$, then we get the next lemma.

Lemma 5.3. We have $t_{a_{g+1}} t_{a_{g+1}^{\prime}}=W_{2}^{g}\left(1, \psi_{i}\right)$ in $\operatorname{Mod}_{g}^{2}$.

Since $t_{a_{g+1}}=1$ and $t_{a_{g+1}^{\prime}}=1$ in $\operatorname{Mod}_{g}$, the word $W_{2}^{g}\left(1, \psi_{i}\right)$ in $\operatorname{Mod}_{g}$ is a positive relator. Therefore, we obtain a genus- $g$ Lefschetz fibration $f_{W_{2}^{g}\left(1, \psi_{i}\right)}$ with two disjoint $(-1)$-sections (and genus- $g$ Lefschetz pencil with two base points corresponding to $W_{2}^{g}\left(1, \psi_{i}\right)$ ). Then, we have the following results which we prove in Section 5B and in Section 5C. 
Theorem 5.4. Suppose that $k=0$. We denote by $F_{n}$ a free group of rank $n$. If $g \geq 2(2 n+1)$, then we have

$$
\pi_{1}\left(X_{W_{2}^{g}\left(1, \psi_{0}\right)}\right) \cong F_{n} .
$$

Theorem 5.5. Suppose that $k>0$. If $g \geq 4(n+l-1)+k$, then we have

$$
\pi_{1}\left(X_{W_{2}^{g}\left(1, \psi_{k}\right)}\right) \cong \Gamma \text {. }
$$

Combining Theorem 5.4 and 5.5, we obtain Theorem 1.2.

5B. Proof of Theorem 5.4. In this section, we prove Theorem 5.4. We begin with a lemma.

Lemma 5.6. Let $r=2 h_{1}+h_{2}-1$. Let $\langle S\rangle$ be the normal closure of the elements of the set $S$ of simple closed curves on $\Sigma_{g}$ defined by

$$
S=\left\{B_{0}, B_{1}, \ldots, B_{g}, D_{0}, D_{1}, \ldots, D_{2 h_{1}}, E_{h_{1}}, A_{4 h_{1}+2}, \ldots, A_{2 r}, a_{r}, a_{r}^{\prime}\right\}
$$

if $g=2 r$, and by

$S=\left\{B_{0}, B_{1}, \ldots, B_{g}, a_{r+1}, a_{r+1}^{\prime}, D_{0}, D_{1}, \ldots, D_{2 h_{1}}, E_{h_{1}}, A_{4 h_{1}+2}, \ldots, A_{2 r+1}, a_{r}, a_{r}^{\prime}\right\}$

if $g=2 r+1$. Then $\pi_{1}\left(\Sigma_{g}\right) /\langle S\rangle$ has a presentation with generators $a_{1}, b_{1}, \ldots, a_{g}, b_{g}$ and with relations

$$
\begin{aligned}
a_{i} a_{g+1-i}=b_{i} a_{g+1-i} b_{g+1-i} a_{g+1-i}^{-1}=1 & & \text { for } 1 \leq i \leq r, \\
a_{2 h_{1}+k}=b_{2 h_{1}+k}=1 & & \text { for } 1 \leq k \leq h_{2}-1, \\
a_{j} a_{2 h_{1}+1-j}=b_{j} a_{2 h_{1}+1-j} b_{2 h_{1}+1-j} a_{2 h_{1}+1-j}^{-1}=1 & & \text { for } 1 \leq j \leq h_{1}, \\
c_{h_{1}}=1 & &
\end{aligned}
$$

if $g=2 r$, and

$$
\begin{aligned}
a_{i} a_{g+1-i}=b_{i} a_{g+1-i} b_{g+1-i} a_{g+1-i}^{-1}=1 & & \text { for } 1 \leq i \leq r, \\
a_{2 h_{1}+k}=b_{2 h_{1}+k}=1 & & \text { for } 1 \leq k \leq h_{2}-1, \\
a_{j} a_{2 h_{1}+1-j}=b_{j} a_{2 h_{1}+1-j} b_{2 h_{1}+1-j} a_{2 h_{1}+1-j}^{-1}=1 & & \text { for } 1 \leq j \leq h_{1}, \\
a_{r+1}=c_{h_{1}}=1 & &
\end{aligned}
$$

if $g=2 r+1$.

Proof. Suppose that $g=2 r$. From the equalities (4) and (5) in Section 2, in $\pi_{1}\left(\Sigma_{g}\right) /\langle S\rangle$ we have

$$
a_{i} a_{g+1-i}=1 .
$$


This gives

$$
\begin{array}{ll}
1=B_{2 i-1}=b_{i} b_{i+1} \cdots b_{g+1-i} c_{g+1-i} & \text { for } 1 \leq i \leq r, \\
1=B_{2 i}=b_{i+1} b_{i+2} \cdots b_{g-i} c_{g-i} & \text { for } 1 \leq i \leq r
\end{array}
$$

in $\pi_{1}\left(\Sigma_{g}\right) /\langle S\rangle$. From these two equalities, we have $b_{i} c_{g-i}^{-1} b_{g+1-i} c_{g+1-i}=1$ for each $1 \leq i \leq r$ and

$$
c_{r}=1 \text {. }
$$

Note that $c_{g+1-i}=b_{g+1-i}^{-1} c_{g-i}\left(a_{g+1-i} b_{g+1-i} a_{g+1-i}^{-1}\right)$ from the equality (1). Therefore, by $b_{i} c_{g-i}^{-1} b_{g+1-i} c_{g+1-i}=1$, we obtain

$$
b_{k} a_{g+1-i} b_{g+1-i} a_{g+1-i}^{-1}=1 .
$$

From $a_{r}=1, A_{l}=1$ for $4 h_{1}+2 \leq l \leq 2 r$ and the equalities (7) and (8), we obtain

$$
a_{2 h_{1}+k}=b_{2 h_{1}+k}=1
$$

for $1 \leq k \leq h_{2}-1$. From $a_{r}^{\prime}=1$ and the equalities (6), (14), (1) and (16), we have

$$
c_{r-1}=c_{2 h_{1}}=1 \text {. }
$$

By $a_{2 h_{1}+1}=1, c_{2 h_{1}}=1$ and the equalities (9), (10) and (11), an argument similar to the proofs of the relations (13) and (15) gives

$$
a_{j} a_{2 h_{1}+1-j}=b_{j} a_{2 h_{1}+1-j} b_{2 h_{1}+1-j} a_{2 h_{1}+1-j}^{-1}=1 \text { and } c_{h_{1}}=1
$$

for $1 \leq j \leq 2 h_{1}$.

From the equalities (13), (14), (15), (16), (17) and (18), we see that $\pi_{1}\left(\Sigma_{g}\right) /\langle S\rangle$ has a presentation with generators $a_{1}, b_{1}, \ldots, a_{g}, b_{g}$ and with relations

$$
\begin{array}{rlrl}
a_{i} a_{g+1-i}=b_{i} a_{g+1-i} b_{g+1-i} a_{g+1-i}^{-1} & =1 & & \text { for } 1 \leq i \leq r, \\
a_{2 h_{1}+k}=b_{2 h_{1}+k}=1 & & \text { for } 1 \leq k \leq h_{2}-1, \\
a_{j} a_{2 h_{1}+1-j}=b_{j} a_{2 h_{1}+1-j} b_{2 h_{1}+1-j} a_{2 h_{1}+1-j}^{-1} & =1 & & \text { for } 1 \leq j \leq h_{1}, \\
c_{g}=c_{r}=c_{r-1}=c_{2 h_{1}}=c_{h_{1}} & =1 . & &
\end{array}
$$

Then by the equalities (1), (16) and (18), we can delete from the above the relations $c_{g}=c_{r}=c_{r-1}=c_{2 h_{1}}=1$. This is our claim.

Suppose now that $g=2 r+1$. Since $a_{r+1}=a_{r+1}^{\prime}=1$ and $a_{r+1}^{\prime}=c_{r} a_{r+1}$, we have $c_{r}=1$. A similar argument as in the case $g=2 r$ shows that $\pi_{1}\left(\Sigma_{g}\right) /\langle S\rangle$ has the desired presentation. This completes the proof.

We can now prove Theorem 5.4. 
Proof of Theorem 5.4. Let $h_{1} \geq n$ and $h_{2}-1 \geq 1$. For simplicity of notation, we write $G$ instead of $\pi_{1}\left(X_{W_{2}^{g}\left(1, \psi_{0}\right)}\right)$.

Suppose that $g=2\left(2 h_{1}+h_{2}-1\right)$ and let $r=2 h_{1}+h_{2}-1$. Note that $G$ has a presentation with generators $a_{1}, b_{1}, \ldots, a_{g}, b_{g}$ and with relations

$$
\begin{aligned}
c_{g} & =1, \\
B_{i} & =1 \quad \text { for } 0 \leq i \leq g, \\
a_{r}=a_{r}^{\prime}=E_{h_{1}} & =1, \\
D_{j}=A_{k} & =1 \quad \text { for } 0 \leq j \leq 2 h_{1}, 4 h_{1}+2 \leq k \leq 4 h_{1}+2 h_{2}-2,
\end{aligned}
$$

$\psi_{0}\left(a_{r}\right)=\psi_{0}\left(a_{r}^{\prime}\right)=\psi_{0}\left(E_{h_{1}}\right)=1$,

$$
\psi_{0}\left(D_{j}\right)=\psi_{0}\left(A_{k}\right)=1 \quad \text { for } 0 \leq j \leq 2 h_{1}, 4 h_{1}+2 \leq k \leq 4 h_{1}+2 h_{2}-2 \text {. }
$$

It is easily seen that, up to conjugation, we have the equalities

$$
\begin{array}{rlrl}
\psi_{0}\left(D_{0}\right) & =a_{h_{1}} \cdots a_{n+2} a_{n+1} D_{0}, & \\
\psi_{0}\left(D_{2 l-1}\right) & =b_{2 h_{1}-l+1}^{-1} a_{h_{1}} \cdots a_{n+2} a_{n+1} D_{2 l-1} & & \text { for } 1 \leq l \leq n, \\
\psi_{0}\left(D_{2 l}\right) & =b_{2 h_{1}-l+1}^{-1} a_{h_{1}} \cdots a_{n+2} a_{n+1} D_{2 l} & & \text { for } 1 \leq l \leq n
\end{array}
$$

in $\pi_{1}\left(\Sigma_{g}\right)$. Thus, by $D_{0}=\psi_{0}\left(D_{0}\right)=D_{j}=\psi_{0}\left(D_{j}\right)=1$ for $1 \leq j \leq 2 h_{1}$, we obtain

$$
b_{2 h_{1}-l+1}=1 \quad \text { for } 1 \leq l \leq n .
$$

Similarly, we have the following equalities (up to conjugation) in $\pi_{1}\left(\Sigma_{g}\right)$ :

$$
\begin{array}{rlrl}
\psi_{0}\left(D_{2 l-1}\right) & =b_{2 h_{1}-l+1}^{-1} a_{h_{1}} \cdots a_{l+1} a_{l} D_{2 l-1} & & \text { for } n+1 \leq l \leq r-1, \\
\psi_{0}\left(D_{2 l}\right) & =b_{2 h_{1}-l+1}^{-1} a_{h_{1}} \cdots a_{l+2} a_{l+1} D_{2 l-1} & & \text { for } n+1 \leq l \leq r-1, \\
\psi_{0}\left(D_{2 h_{1}-1}\right) & =b_{h_{1}+1}^{-1} a_{h_{1}} D_{2 h_{1}-1}, & \\
\psi_{0}\left(D_{2 h_{1}}\right) & =b_{h_{1}+1}^{-1} B_{2 h_{1}} . & &
\end{array}
$$

By $D_{j}=1$ for $1 \leq j \leq 2 h_{1}$ and $\psi_{0}\left(D_{2 l-1}\right)=\psi_{0}\left(D_{2 l}\right)=1$ for $n+1 \leq l \leq h_{1}$, we obtain

$$
a_{l}=1 \text { for } n+1 \leq l \leq h_{1} .
$$

Moreover, by $\psi_{0}\left(D_{2 l}\right)=\psi_{0}\left(D_{2 l+1}\right)=\psi_{0}\left(D_{2 h_{1}}\right)=1$ for $n+1 \leq l \leq h_{1}-1$, we have

$$
b_{2 h_{1}-l+1}=1 \text { for } n+1 \leq l \leq h_{1} .
$$

Here, since $\psi_{0}\left(a_{r}\right)=a_{r}, \psi_{0}\left(a_{r}^{\prime}\right)=a_{r}^{\prime}, \psi_{0}\left(E_{h_{1}}\right)=E_{h_{1}}$ and $\psi_{0}\left(A_{k}\right)=A_{k}$ in $\pi_{1}\left(\Sigma_{g}\right)$ for each $4 h_{1}+2 \leq k \leq 4 h_{1}+2 h_{2}-2$, we can delete the relations $\psi_{0}\left(a_{r}\right)=1$, $\psi_{0}\left(a_{r}^{\prime}\right)=1, \psi_{0}\left(E_{h_{1}}\right)=1$ and $\psi_{0}\left(A_{K}\right)=1$ from the above presentation of $G$. 
From the above arguments and Lemma 5.6, we see that $G$ has a presentation with generators $a_{1}, b_{1}, \ldots, a_{g}, b_{g}$ and with relations

$$
\begin{array}{rlrl}
a_{i} a_{g+1-i}=b_{i} a_{g+1-i} b_{g+1-i} a_{g+1-i}^{-1} & & \text { for } 1 \leq i \leq r, \\
a_{2 h_{1}+k}=b_{2 h_{1}+k} & =1 & & \text { for } 1 \leq k \leq h_{2}-1, \\
a_{j} a_{2 h_{1}+1-j}=b_{j} a_{2 h_{1}+1-j} b_{2 h_{1}+1-j} a_{2 h_{1}+1-j}^{-1} & =1 & & \text { for } 1 \leq j \leq h_{1}, \\
c_{h_{1}} & =1, & \\
a_{n+1}=a_{n+2}=\cdots=a_{h_{1}} & =1, & \\
b_{h_{1}}=b_{h_{1}+1}=\cdots=b_{2 h_{1}} & =1 . &
\end{array}
$$

It is easily shown that this is a presentation of the free group of rank $n$ with free basis $a_{1}, \ldots, a_{n}$, that is, $G$ is isomorphic to $F_{n}$.

The proof for $g=2 r+1$ is similar. This completes the proof of Theorem 5.4.

5C. Proof of Theorem 5.5. We now prove Theorem 5.5. The proof is inspired by [Korkmaz 2009] and that of Proposition 13 in [Akhmedov and Ozbagci 2013]. For simplicity, we write $G^{\prime}$ instead of $\pi_{1}\left(X_{W_{2}^{g}\left(1, \psi_{1}\right)}\right)$.

Proof of Theorem 5.5. Suppose that $g=2\left(2 h_{1}+h_{2}-1\right)$. Since $R_{1}$ intersects $a_{2 h_{1}+h_{2}-1}$ at one point and does not intersect $A_{j}$ for $j=4 h_{1}+2, \ldots, 4 h_{1}+2 h_{2}-2$, and $a_{2 h_{1}+h_{2}-1}$ is disjoint from $a_{n+1}, \ldots, a_{h_{1}}, b_{h_{1}+1}, \ldots, b_{2 h_{1}}$ and $R_{2}, \ldots, R_{k}$, we see that in $\pi_{1}\left(\Sigma_{g}\right)$, up to conjugation,

$$
\psi_{k}\left(a_{2 h_{1}+h_{2}-1}\right)=t_{R_{1}}\left(a_{2 h_{1}+h_{2}-1}\right)=a_{2 h_{1}+h_{2}-1} R_{1}^{\epsilon},
$$

where $\epsilon$ is equal to 1 or -1 . Since $a_{2 h_{1}+h_{2}-1}=1$ in $G^{\prime}$, we may replace the relator $\psi_{k}\left(a_{2 h_{1}+h_{2}-1}\right)=1$ by $R_{1}=1$.

Let $c$ be an element of the set of the vanishing cycles of $f_{W_{2}^{g}\left(1, \psi_{k}\right)}$. If $R_{1}$ is disjoint from $\psi_{k-1}(c)$, then we have $\psi_{k}(c)=t_{R_{1}}\left(\psi_{k-1}(c)\right)=\psi_{k-1}(c)$. If $R_{1}$ intersects $\psi_{k-1}(c)$ at $t$ points, then it is easily seen that there are elements $x_{1}, \ldots, x_{t+1}$ in $\pi_{1}\left(\Sigma_{g}\right)$ such that $\psi_{k-1}(c)=x_{1} x_{2} \cdots x_{t+1}$ and that

$$
t_{R_{1}}\left(\psi_{k-1}(c)\right)=x_{1} R_{1}^{\zeta_{1}} x_{2} R_{1}^{\zeta_{2}} \cdots x_{t} R_{1}^{\zeta t} x_{t+1}
$$

(up to conjugacy), where each $\zeta_{s}$ is equal to 1 or -1 . From $R_{1}=1$, we obtain $\psi_{k}(c)=t_{R_{1}}\left(\psi_{k-1}(c)\right)=\psi_{k-1}(c)$ in $G^{\prime}$. Therefore, we may replace the relator $\psi_{k}(c)=1$ by $\psi_{k-1}(c)=1$.

By repeating this argument for each $i=k-1, \ldots, 1$, we see that we may replace the relators $\psi_{k}\left(A_{4 h_{1}+2 h_{2}-(k+1-i)}\right)=1$ and $\psi_{k}(c)=1$ by $R_{k+1-i}=1$ and $\psi_{0}(c)=1$, respectively. In particular, since for each $j=4 h_{1}+2, \ldots, 4 h_{1}+2 h_{2}-2$, $a_{2 h_{1}+h_{2}-1}=1$ and $A_{j}=1$ in $G^{\prime}$ and $a_{2 h_{1}+h_{2}-1}=\psi_{0}\left(a_{2 h_{1}+h_{2}-1}\right)$ and $A_{j}=\psi_{0}\left(A_{j}\right)$ in $\pi_{1}\left(\Sigma_{g}\right)$ (up to conjugation), we can delete the relators $\psi_{k}\left(a_{2 h_{1}+h_{2}-1}\right)=1$ and 
$\psi_{k}\left(A_{j}\right)=1$. Therefore, from the proof of Theorem 5.4, we see that $G^{\prime}$ has a presentation with generators $a_{1}, b_{1}, \ldots, a_{g}, b_{g}$ and with relations

$$
\begin{array}{rlrl}
a_{i} a_{g+1-i}=b_{i} a_{g+1-i} b_{g+1-i} a_{g+1-i}^{-1} & & \text { for } 1 \leq i \leq r, \\
a_{2 h_{1}+k}=b_{2 h_{1}+k} & =1 & & \text { for } 1 \leq k \leq h_{2}-1, \\
a_{j} a_{2 h_{1}+1-j}=b_{j} a_{2 h_{1}+1-j} b_{2 h_{1}+1-j} a_{2 h_{1}+1-j}^{-1} & =1 & \text { for } 1 \leq j \leq h_{1}, \\
c_{h_{1}} & =1, & \\
a_{n+1}=a_{n+2}=\cdots=a_{h_{1}} & =1, & \\
b_{h_{1}}=b_{h_{1}+1}=\cdots=b_{2 h_{1}} & =1, & \\
R_{1}=R_{2}=\cdots=R_{k} & =1 . &
\end{array}
$$

We note that the element $\left[R_{i}\right] \in \pi_{1}\left(\Sigma_{g}\right)$ is contained in the subgroup generated by $a_{1}, b_{1}, \ldots, a_{h_{1}}, b_{h_{1}}$ and $a_{2 h_{1}+1}, b_{2 h_{1}+1}, \ldots, a_{2 h_{1}+h_{2}-1}, b_{2 h_{1}+h_{2}-1}$. Since from this presentation, we see that $a_{s}=1$ for $s=n+1, \ldots, h_{1}, 2 h_{1}+1, \ldots, 2 h_{1}+h_{2}-1$ and $b_{j}=1$ for $j=1, \ldots, h_{1}, 2 h_{1}+1, \ldots, 2 h_{1}+h_{2}-1$, we get a word representing the element $r_{i}$ by Proposition 5.2. Therefore, $G^{\prime}$ is isomorphic to $\Gamma$.

A similar argument works for $g=2\left(2 h_{1}+h_{2}-1\right)+1$. Since $f_{W_{2}^{g}\left(1, \psi_{k}\right)}$ has at least two disjoint $(-1)$-sections, by blowing down one of them we obtain the required genus- $g$ Lefschetz pencil. This completes the proof of Theorem 5.5 and therefore, as discussed in Section 5A, also of Theorem 1.2.

\section{Construction of a lift of Gurtas' positive relator}

In this section, we prove Proposition 5.1 and give a lift to $\operatorname{Mod}_{g}^{2}$ of the positive relator in $\operatorname{Mod}_{g}$ given by Gurtas [2004].

6A. Outline of the proof of Lemma 3.4. We now give an outline of the proof of Lemma 3.4, which is needed to prove Proposition 5.1.

Outline of the proof of Lemma 3.4. We define $\Delta_{0}=\bar{\Delta}_{0}=1$. Moreover, for each $k=1, \ldots, 2 g+1$, we define $\Delta_{k}$ and $\bar{\Delta}_{k}$ to be the words

$$
\Delta_{k}=t_{A_{1}} t_{A_{2}} \cdots t_{A_{k}} \quad \text { and } \quad \bar{\Delta}_{k}=t_{A_{k}} \cdots t_{A_{2}} t_{A_{1}} .
$$

For each $k=0,1, \ldots, g$, the words $\beta_{k}$ and $\beta$ are defined by

$$
\beta_{k}=\bar{\Delta}_{k} \Delta_{2 g+1-k} \Delta_{2 g-k}^{-1} \bar{\Delta}_{k}^{-1} \text { and } \beta=\bar{\Delta}_{g}^{g+1} .
$$

Then by applying the argument from Section 2 of [Korkmaz 2001] with $\sigma_{i}$ (which is the standard generator of the braid group $\mathrm{B}_{2 g+2}$ on $2 g+2$ strings) replaced by $t_{A_{i}}$, we have the relation

$$
\beta_{0} \beta_{1} \beta_{2} \cdots \beta_{g} \beta^{2}=\Delta_{2 g+1} \Delta_{2 g} \cdots \Delta_{3} \Delta_{2} \Delta_{1} .
$$


It is easy to check that $\bar{\Delta}_{k} \Delta_{2 g-k}\left(A_{2 g+1-k}\right)=B_{k}$. This gives

$$
t_{B_{k}}=\left(\bar{\Delta}_{k} \Delta_{2 g-k}\right) t_{A_{2 g+1-k}}\left(\bar{\Delta}_{k} \Delta_{2 g-k}\right)^{-1}=\bar{\Delta}_{k} \Delta_{2 g+1-k} \Delta_{2 g-k}^{-1} \bar{\Delta}_{k}^{-1}=\beta_{k} .
$$

Therefore, from the relation (19), we have

$$
t_{B_{0}} t_{B_{1}} t_{B_{2}} \cdots t_{B_{g}}\left(\bar{\Delta}_{g}\right)^{2 g+2}=\Delta_{2 g+1} \Delta_{2 g} \cdots \Delta_{3} \Delta_{2} \Delta_{1} .
$$

Using the chain relations $\bar{\Delta}_{g}^{2 g+2}=t_{c_{r}}$ when $g=2 r$ and $\bar{\Delta}_{g}^{g+1}=t_{a_{r+1}} t_{a_{r+1}^{\prime}}$ when $g=2 r+1$, we have

$$
\Delta_{2 g+1} \Delta_{2 g} \cdots \Delta_{3} \Delta_{2} \Delta_{1}= \begin{cases}t_{B_{0}} t_{B_{1}} t_{B_{2}} \cdots t_{B_{g}} t_{c_{r}} & \text { for } g=2 r, \\ t_{B_{0}} t_{B_{1}} t_{B_{2}} \cdots t_{B_{g}} t_{a_{r+1}} t_{a_{r+1}^{\prime}} & \text { for } g=2 r+1 .\end{cases}
$$

If we prove that $t_{a_{g+1}} t_{a_{g+1}^{\prime}}=\left(\Delta_{2 g+1} \Delta_{2 g} \cdots \Delta_{3} \Delta_{2} \Delta_{1}\right)^{2}$ in $\operatorname{Mod}_{g}^{2}$, the assertion follows. Note that the chain relation $\Delta_{2 g+1}^{2 g+2}=t_{a_{g+1}} t_{a_{g+1}^{\prime}}$, and $t_{A_{k}} \Delta_{m}=\Delta_{m} t_{A_{k-1}}$ if $1<k \leq m$ (see [Korkmaz 2001, Lemma 2.1(a)]), hold in $\operatorname{Mod}_{g}^{2}$. Then we have

$$
\begin{aligned}
\Delta_{2 g+1}^{2 g+2} & =\Delta_{2 g+1} \Delta_{2 g} t_{A_{2 g+1}} \Delta_{2 g+1} \Delta_{2 g+1}^{2 g-1} \\
& =\Delta_{2 g+1} \Delta_{2 g} \Delta_{2 g+1} t_{A_{2 g}} \Delta_{2 g+1}^{2 g-1} \\
& =\Delta_{2 g+1} \Delta_{2 g} \Delta_{2 g-1}\left(t_{A_{2 g}} t_{A_{2 g+1}}\right) t_{A_{2 g}} \Delta_{2 g+1}^{2 g-1} \\
& =\Delta_{2 g+1} \Delta_{2 g} \Delta_{2 g-1} \Delta_{2 g+1}\left(t_{A_{2 g-1}} t_{A_{2 g}}\right) t_{A_{2 g-1}} \Delta_{2 g+1}^{2 g-2} \\
& =\Delta_{2 g+1} \Delta_{2 g} \Delta_{2 g-1} \Delta_{2 g-2}\left(t_{A_{2 g-1}} t_{A_{2 g}} t_{A_{2 g+1}}\right)\left(t_{A_{2 g-1}} t_{A_{2 g}}\right) t_{A_{2 g-1}} \Delta_{2 g+1}^{2 g-2} \\
& \quad \vdots \\
& =\Delta_{2 g+1} \Delta_{2 g} \cdots \Delta_{1}\left(t_{A_{2}} t_{A_{3}} \cdots t_{A_{2 g+1}}\right)\left(t_{A_{2}} t_{A_{3}} \cdots t_{A_{2 g}}\right) \cdots\left(t_{A_{2}} t_{A_{3}}\right) t_{A_{2}} \Delta_{2 g+1} \\
& =\Delta_{2 g+1} \Delta_{2 g} \cdots \Delta_{1} \Delta_{2 g+1} \Delta_{2 g} \cdots \Delta_{1},
\end{aligned}
$$

and the proof is complete.

6B. Proof of Proposition 5.1. In this section, we prove Proposition 6.1 instead of Proposition 5.1. Note that if we set $g=r$ in the notation of Proposition 6.1 and consider an embedding $\Sigma_{r}^{2} \hookrightarrow \Sigma_{g}^{2}$ (resp. $\left.\Sigma_{r}^{1} \hookrightarrow \Sigma_{g}^{2}\right)$ mapping $\left(a_{r+1}, a_{r+1}^{\prime}\right)$ (resp. $\left.a_{r+1}\right)$ in Proposition 6.1 to $\left(a_{r+1}, a_{r+1}^{\prime}\right)$ (resp. $\left.c_{r}\right)$ in Proposition 5.1, then we get Proposition 5.1. Therefore, it is sufficient to prove Proposition 6.1.

Proposition 6.1. Let $\Sigma_{g}^{2}$ (resp. $\Sigma_{g}^{1}$ ) be the compact oriented surface of genus $g$ with two boundary components (resp. one boundary component) obtained from $\Sigma_{g}$ by removing two disjoint open disks (resp. one open disk). Let $a_{g+1}, a_{g+1}^{\prime}=c_{g} a_{g+1}$ (resp. $\left.a_{g+1}\right)$ be the boundary curves of $\Sigma_{g}^{2}$ (resp. the boundary curve of $\Sigma_{g}^{1}$ ). Then 
the relations

(21)

$$
\begin{gathered}
t_{a_{g+1}} t_{a_{g+1}^{\prime}}=t_{E_{h_{1}}} t_{A_{4 h_{1}+2}} \cdots t_{A_{2 g}} t_{a_{g}} t_{a_{g}^{\prime}} t_{A_{2 g}} \cdots t_{A_{4 h_{1}+2}} t_{E_{h_{1}}} \\
\cdot t_{A_{2 g+1}} t_{A_{2 g}} \cdots t_{A_{4 h_{1}+2}} \cdot\left(t_{D_{0}} t_{D_{1}} \cdots t_{D_{2 h_{1}}}\right)^{2} \cdot t_{A_{4 h_{1}+2}} \cdots t_{A_{2 g}} t_{A_{2 g}+1}, \\
t_{a_{g+1}}=t_{E_{h_{1}}} t_{A_{4 h_{1}+2}} \cdots t_{A_{2 g}} t_{a_{g}} t_{a_{g}^{\prime}} t_{A_{2 g}} \cdots t_{A_{4 h_{1}+2}} t_{E_{h_{1}}} \\
\cdot t_{a_{g}^{\prime}} t_{A_{2 g}} \cdots t_{A_{4 h_{1}+2}} \cdot\left(t_{D_{0}} t_{D_{1}} \cdots t_{D_{2 h_{1}}}\right)^{2} \cdot t_{A_{4 h_{1}+2}} \cdots t_{A_{2 g}} t_{a_{g}^{\prime}}
\end{gathered}
$$

hold in $\operatorname{Mod}_{g}^{2}$ and $\operatorname{Mod}_{g}^{1}$, respectively.

In order to prove Proposition 6.1, we prepare Lemma 6.2 and Proposition 6.3.

Lemma 6.2. Suppose that $g=2 r$. In the notation of Lemma 3.4, let $c_{r}^{\prime}$ be the separating simple closed curve defined by $a_{g+1}\left(b_{r+1} \cdots b_{g}\right) a_{g+1}^{\prime}\left(b_{r+1} \cdots b_{g}\right)^{-1} c_{r}$ (cf. Figure $6(a)$ ). We modify $\Sigma_{g}^{2}$ and $B_{0}, \ldots, B_{g}, c_{r}, c_{r}^{\prime}$ by isotopy as shown in Figure 6(b) and (c). Then in $\operatorname{Mod}_{g}^{2}$, the following relation holds:

$$
t_{a_{g+1}} t_{a_{g+1}^{\prime}}=t_{c_{r}} t_{c_{r}^{\prime}}\left(t_{B_{0}} t_{B_{1}} \cdots t_{B_{g}}\right)^{2}
$$

Proof. It is easily seen that for each $i=1, \ldots, g$, we have

$$
\Delta_{2 g+1} \cdots \Delta_{2} \Delta_{1}\left(A_{i}\right)=A_{2 g+2-i}
$$

This gives the relation

$$
\Delta_{2 g+1} \cdots \Delta_{2} \Delta_{1} t_{A_{i}}=t_{A_{2 g+i}} \Delta_{2 g+1} \cdots \Delta_{2} \Delta_{1}
$$

for each $i=1, \ldots, 2 r$. Therefore, we have

$$
\Delta_{2 g+1} \cdots \Delta_{2} \Delta_{1}\left(\bar{\Delta}_{g}\right)^{-(2 g+2)}=\left(t_{A_{g+2}} \cdots t_{A_{2 g+1}}\right)^{-(2 g+2)} \Delta_{2 g+1} \cdots \Delta_{2} \Delta_{1} \text {. }
$$
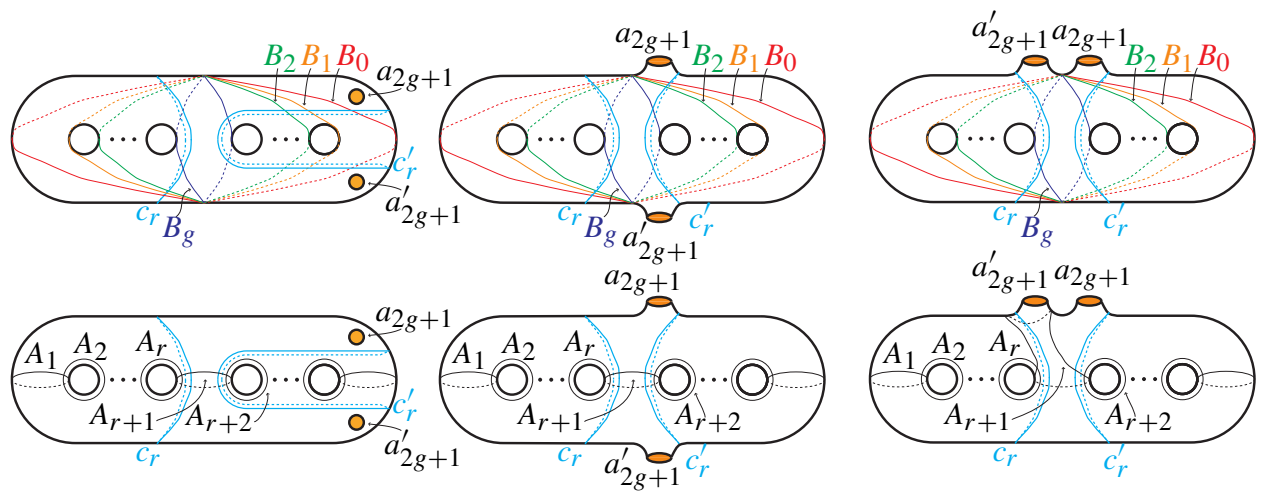

(a)

(b)

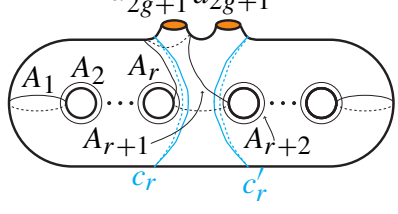

(c)

Figure 6. Modified surface $\Sigma_{g}^{2}$ and curves $B_{0}, \ldots, B_{g}, c_{r}, c_{r}^{\prime}$. 
Since

$$
t_{B_{0}} t_{B_{1}} t_{B_{2}} \cdots t_{B_{g}}\left(\bar{\Delta}_{g}\right)^{2 g+2}=\Delta_{2 g+1} \cdots \Delta_{2} \Delta_{1}\left(=t_{B_{0}} t_{B_{1}} t_{B_{2}} \cdots t_{B_{g}} t_{c_{r}}\right)
$$

from the proof of Lemma 3.4, we have

$$
\begin{aligned}
\left(t_{A_{g+2}} \cdots t_{A_{2 g+1}}\right)^{2 g+2} t_{B_{0}} t_{B_{1}} t_{B_{2}} \cdots t_{B_{g}} & =\Delta_{2 g+1} \cdots \Delta_{2} \Delta_{1} \\
( & \left.=t_{B_{0}} t_{B_{1}} t_{B_{2}} \cdots t_{B_{g}} t_{c_{r}}\right) .
\end{aligned}
$$

By the chain relation, we obtain $t_{c_{r}^{\prime}}=\left(t_{A_{g+2}} \cdots t_{A_{2 g+1}}\right)^{2 g+2}$. Therefore,

$$
t_{a_{g+1}} t_{a_{g+1}^{\prime}}=t_{c_{r}^{\prime}} t_{B_{0}} t_{B_{1}} \cdots t_{B_{g}} \cdot t_{B_{0}} t_{B_{1}} \cdots t_{B_{g}} t_{c_{r}}
$$

follows by Lemma 3.4. By conjugation by $t_{c_{r}}$, we have

$$
t_{a_{g+1}} t_{a_{g+1}^{\prime}}=t_{c_{r}} t_{c_{r}^{\prime}}\left(t_{B_{0}} t_{B_{1}} \cdots t_{B_{g}}\right)^{2} \text {. }
$$

Proposition 6.3 was shown by Hamada [ $\geq 2016]$ based on the argument of [Tanaka 2012]. Its statement concerns $a_{0}^{\prime}$, a null-homotopic simple closed curve in $\Sigma_{g}$ defined by $a_{0}^{\prime}=c_{0} a_{0}$.

Proposition 6.3 [Hamada $\geq 2016$ ]. Let $\Sigma_{g}^{4}$ be the compact oriented surface of genus $g$ with four boundary components obtained from $\Sigma_{g}$ by removing four disjoint open disks surrounded by $a_{0}, a_{0}^{\prime}, a_{g+1}$ and $a_{g+1}^{\prime}$. Then the following relation in $\operatorname{Mod}_{g}^{4}$ holds:

$$
t_{a_{0}} t_{a_{0}^{\prime}} t_{a_{g+1}} t_{a_{g+1}^{\prime}}=t_{A_{2 g+1}} \cdots t_{A_{2}} t_{a_{1}} t_{a_{1}^{\prime}} t_{A_{2}} \cdots t_{A_{2 g+1}} \cdot t_{A_{1}} \cdots t_{A_{2 g}} t_{a_{g}} t_{a_{g}^{\prime}} t_{A_{2 g}} \cdots t_{A_{1}} .
$$

Proof. The proof is by induction on the genus.

Suppose that $g=1$. The four-holed torus relation,

$$
t_{a_{0}} t_{a_{0}^{\prime}} t_{a_{2}} t_{a_{2}^{\prime}}=\left(t_{A_{1}} t_{A_{3}} t_{A_{2}} t_{a_{1}} t_{a_{1}^{\prime}} t_{A_{2}}\right)^{2},
$$

was constructed by Korkmaz and Ozbagci [2008, Section 3.4]. Since $a_{0}, a_{0}^{\prime}, a_{2}, a_{2}^{\prime}$ are disjoint from $A_{1}$ and $A_{1}$ is disjoint from $A_{3}$, by conjugation by $t_{A_{1}}$, we have

$$
\begin{aligned}
t_{a_{0}} t_{a_{0}^{\prime}} t_{a_{2}} t_{a_{2}^{\prime}} & =t_{A_{3}} t_{A_{2}} t_{a_{1}} t_{a_{1}^{\prime}} t_{A_{2}} t_{A_{1}} \cdot t_{A_{3}} t_{A_{2}} t_{a_{1}} t_{a_{1}^{\prime}} t_{A_{2}} t_{A_{1}} \\
& =t_{A_{3}} t_{A_{2}} t_{a_{1}} t_{a_{1}^{\prime}} t_{A_{2}} t_{A_{3}} \cdot t_{A_{1}} t_{A_{2}} t_{a_{1}} t_{a_{1}^{\prime}} t_{A_{2}} t_{A_{1}} .
\end{aligned}
$$

Hence, the conclusion of the proposition holds for $g=1$.

Next we assume, inductively, that the relation holds in $\operatorname{Mod}_{g-1}^{4}$. Since then $a_{0}, a_{0}^{\prime}, a_{g}, a_{g}^{\prime}$ are disjoint from $A_{1}, \ldots, A_{2 g-1}$, we have the relation

$$
t_{a_{0}} t_{a_{0}^{\prime}} t_{a_{g}} t_{a_{g}^{\prime}}=t_{A_{2 g-2}} \cdots t_{A_{1}} \cdot t_{A_{2 g-1}} \cdots t_{A_{2}} t_{a_{1}} t_{a_{1}^{\prime}} t_{A_{2}} \cdots t_{A_{2 g-1}} \cdot t_{A_{1}} \cdots t_{A_{2 g-2}} t_{a_{g-1}} t_{a_{g-1}}^{\prime}
$$

in $\operatorname{Mod}_{g}^{4}$ by conjugation by $t_{A_{2 g-2}} \cdots t_{A_{1}}$. Since $a_{g-1}, a_{g-1}^{\prime}, a_{g+1}, a_{g+1}^{\prime}$ are disjoint from $A_{2 g-1}, A_{2 g}, A_{2 g+1}, a_{g}, a_{g}^{\prime}$, by the four-holed torus relation

$$
t_{a_{g-1}} t_{a_{g-1}^{\prime}} t_{a_{g+1}} t_{a_{g+1}^{\prime}}^{\prime}=\left(t_{A_{2 g-1}} t_{A_{2 g+1}} t_{A_{2 g}} t_{a_{g}} t_{a_{g}^{\prime}} t_{A_{2 g}}\right)^{2}
$$


and conjugation by $t_{A_{2 g-1}} t_{A_{2 g+1}} t_{A_{2 g}}$, we have the relation

$$
t_{a_{g}}^{-1} t_{a_{g}^{\prime}}^{-1} t_{a_{g+1}} t_{a_{g+1}^{\prime}}^{\prime}=t_{a_{g-1}^{\prime}}^{-1} t_{a_{g-1}}^{-1} t_{A_{2 g}} t_{A_{2 g-1}} t_{A_{2 g+1}} t_{A_{2 g}} t_{a_{g}} t_{a_{g}^{\prime}} t_{A_{2 g}} t_{A_{2 g-1}} t_{A_{2 g+1}} t_{A_{2 g}} .
$$

By combining these relations, we have

$$
\begin{aligned}
t_{a_{0}} t_{a_{0}^{\prime}} t_{a_{g+1}} t_{a_{g+1}^{\prime}}=t_{A_{2 g-2}} \cdots t_{A_{1}} \cdot t_{A_{2 g-1}} & \cdots t_{A_{2}} t_{a_{1}} t_{a_{1}^{\prime}} t_{A_{2}} \cdots t_{A_{2 g-1}} \cdot t_{A_{1}} \cdots t_{A_{2 g-2}} \\
\cdot & t_{A_{2 g}} t_{A_{2 g-1}} t_{A_{2 g+1}} t_{A_{2 g}} \cdot t_{a_{g}} t_{a_{g}^{\prime}} t_{A_{2 g}} t_{A_{2 g-1}} t_{A_{2 g+1}} t_{A_{2 g}} .
\end{aligned}
$$

Note that $A_{1}, \ldots, A_{2 g+1}$ are disjoint from $a_{0}, a_{0}^{\prime}, a_{g+1}, a_{g+1}^{\prime}$. Moreover, $A_{2 g}$ and $A_{2 g+1}$ are disjoint from $A_{1}, \ldots, A_{2 g-2}$ and $A_{1}, \ldots, A_{2 g-1}$, respectively. Therefore, by conjugation by $t_{A_{2 g-2}} \cdots t_{A_{1}}$ and $t_{A_{2 g+1}} t_{A_{2 g}}$, we have

$$
\begin{aligned}
t_{a_{0}} t_{a_{0}^{\prime}} t_{a_{g+1}} t_{a_{g+1}^{\prime}}^{\prime}=t_{A_{2 g-2}} \cdots t_{A_{1}} \cdot t_{A_{2 g-1}} & \cdots t_{A_{2}} t_{a_{1}} t_{a_{1}^{\prime}} t_{A_{2}} \cdots t_{A_{2 g-1}} \cdot t_{A_{1}} \cdots t_{A_{2 g-2}} \\
\cdot & t_{A_{2 g}} t_{A_{2 g-1}} t_{A_{2 g+1}} t_{A_{2 g}} \cdot t_{a_{g}} t_{a_{g}^{\prime}} t_{A_{2 g}} t_{A_{2 g-1}} t_{A_{2 g+1}} t_{A_{2 g}} \\
=t_{A_{2 g+1}} t_{A_{2 g}} \cdot t_{A_{2 g-1}} \cdots & t_{A_{2}} t_{a_{1}} t_{a_{1}^{\prime}} t_{A_{2}} \cdots t_{A_{2 g-1}} \cdot t_{A_{2 g}} t_{A_{2 g+1}} \cdot t_{A_{1}} \cdots t_{A_{2 g-2}} \\
\cdot & t_{A_{2 g-1}} t_{A_{2 g}} \cdot t_{a_{g}} t_{a_{g}^{\prime}} t_{A_{2 g}} t_{A_{2 g-1}} t_{A_{2 g-2}} \cdots t_{A_{1}} .
\end{aligned}
$$

This completes the proof of Proposition 6.3.

We now prove Proposition 6.1.

Proof of Proposition 6.1. Let $c_{h_{1}}^{\prime}$ be the separating simple closed curve as shown in Figure 7. By Lemma 6.2 and Proposition 6.3, we have

$$
\begin{aligned}
& t_{a_{h_{1}+1}} t_{a_{h_{1}+1}^{\prime}}^{\prime}=t_{c_{h_{1}}} t_{c_{h_{1}}^{\prime}}\left(t_{D_{0}} t_{D_{1}} \cdots t_{D_{2 h_{1}}}\right)^{2} \\
& t_{c_{h_{1}} t_{c_{h_{1}}^{\prime}} t_{a_{g+1}} t_{a_{g+1}^{\prime}}^{\prime}}=t_{a_{g} t_{A_{2 g}} \cdots t_{A_{4 h_{1}+2}} t_{E_{h_{1}}} t_{E_{h_{1}}} t_{A_{4 h_{1}+2}} \cdots t_{A_{2 g}} t_{a_{g}^{\prime}}} \\
& \cdot t_{A_{2 g+1}} \cdots t_{A_{4 h_{1}+2} t_{a_{h_{1}+1}} t_{a_{h_{1}+1}^{\prime}} t_{A_{4 h_{1}+2}} \cdots t_{A_{2 g+1}}} .
\end{aligned}
$$

Since $c_{h_{1}}$ and $c_{h_{1}}^{\prime}$ are disjoint from $A_{2 h_{1}+2}, \ldots, A_{2 g}, E_{h_{1}}, a_{h_{1}+1}, a_{h_{1}+1}^{\prime}$, it follows that

$$
\begin{aligned}
t_{c_{h_{1}}^{\prime}}^{-1} t_{h_{1}}^{-1} \cdot t_{a_{h_{1}+1}} t_{a_{h_{1}+1}^{\prime}}^{\prime}= & \left(t_{D_{0}} t_{D_{1}} \cdots t_{D_{2 h_{1}}}\right)^{2}, \\
t_{a_{g+1}} t_{a_{g+1}}^{\prime}= & t_{a_{g}} t_{A_{2 g}} \cdots t_{A_{4 h_{1}+2}} t_{E_{h_{1}}} t_{E_{h_{1}}} t_{A_{4 h_{1}+2}} \cdots t_{A_{2 g}} t_{a_{g}^{\prime}} \\
& \quad t_{A_{2 g}+1} \cdots t_{A_{4 h_{1}+2}+2} \cdot t_{c_{h_{1}}^{\prime}}^{-1} t_{c_{h_{1}}}^{-1} \cdot t_{a_{h_{1}+1} t_{a_{h_{1}+1}}} \cdot t_{A_{4 h_{1}+2}} \cdots t_{A_{2 g+1}} .
\end{aligned}
$$

Combining these relations gives the relation (21) in Proposition 6.1.

In $\Sigma_{g}^{1}, A_{2 g+1}$ is homotopic to $a_{g}^{\prime}$, and (22) follows, completing the proof. 


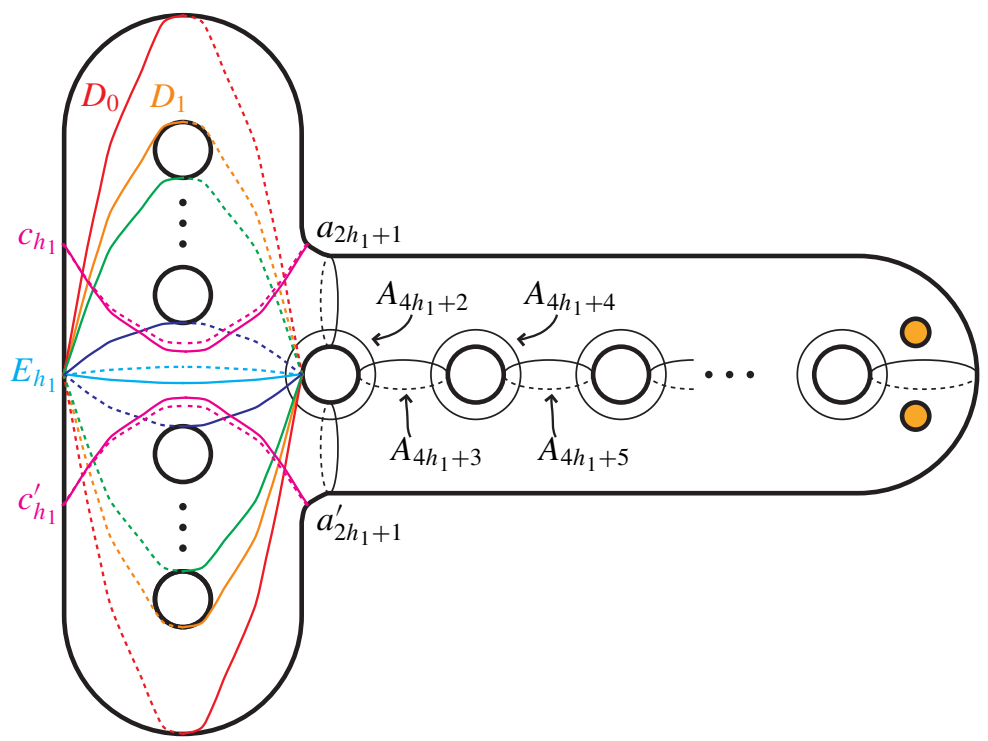

Figure 7. The curve $c_{h_{1}}^{\prime}$ on $\Sigma_{g}^{2}$.

6C. A lift of Gurtas' positive relator. Since $a_{g+1}$ and $a_{g+1}^{\prime}$ are null-homotopic in $\Sigma_{g}$, we have $t_{a_{g+1}}=t_{a_{g+1}^{\prime}}=1$ in $\operatorname{Mod}_{g}$, so the relation in Proposition 6.1 is a positive relator in $\operatorname{Mod}_{g}$. Then we note that $A_{2 g+1}$ and $a_{g}^{\prime}$ are homotopic to $a_{g}$. On the other hand, Gurtas [2004] gave the positive relator

$$
\left(t_{A_{4 h_{1}+2}} \cdots t_{A_{2 g}} t_{a_{g}} t_{a_{g}} t_{A_{2 g}} \cdots t_{A_{4 h_{1}+2}} t_{D_{0}} t_{D_{1}} \cdots t_{D_{2 h_{1}}} t_{E_{h_{1}}}\right)^{2}=1 .
$$

in $\operatorname{Mod}_{g}$. Using the following theorem of Kas [1980] and Matsumoto [1996], we show that the relation in Proposition 6.1 gives a lift of Gurtas' positive relator in $\operatorname{Mod}_{g}$ to $\operatorname{Mod}_{g}^{2}$.

Theorem 6.4 [Kas 1980; Matsumoto 1996]. If $g \geq 2$, then the isomorphism class of a Lefschetz fibration is determined by a positive relator modulo simultaneous conjugations

$$
t_{v_{n}} \cdots t_{v_{2}} t_{v_{1}} \sim t_{\phi\left(v_{n}\right)} \cdots t_{\phi\left(v_{2}\right)} t_{\phi\left(v_{1}\right)} \quad \text { for any } \phi \in \Gamma_{g}
$$

and elementary transformations

$$
\begin{aligned}
& t_{v_{n}} \cdots t_{v_{i+2}} t_{v_{i+1}} t_{v_{i}} t_{v_{i-1}} t_{v_{i-2}} \cdots t_{v_{1}} \sim t_{v_{n}} \cdots t_{v_{i+2}} t_{v_{i}} t_{t_{v_{i}}\left(v_{i+1}\right)} t_{v_{i-1}} t_{v_{i-2}} \cdots t_{v_{1}}, \\
& t_{v_{n}} \cdots t_{v_{i+2}} t_{v_{i+1}} t_{v_{i}} t_{v_{i-1}} t_{v_{i-2}} \cdots t_{v_{1}} \sim t_{v_{n}} \cdots t_{v_{i+2}} t_{v_{i+1}} t_{t_{v_{i}}\left(v_{i-1}\right)} t_{v_{i}} t_{v_{i-2}} \cdots t_{v_{1}} .
\end{aligned}
$$

The aim of this section is to prove the following proposition. This proposition applied to Proposition 6.1 gives the above mentioned lift. 
Proposition 6.5. In $\operatorname{Mod}_{g}$, the following relation holds:

$$
\begin{aligned}
t_{E_{h_{1}}} t_{A_{4 h_{1}+2}} \cdots t_{A_{2 g}} t_{a_{g}} t_{a_{g}} t_{A_{2 g}} \cdots t_{A_{4 h_{1}+2}} t_{E_{h_{1}}} & \\
\cdot t_{a_{g}} t_{A_{2 g}} \cdots & t_{A_{4 h_{1}+2}} \cdot\left(t_{D_{0}} t_{D_{1}} \cdots t_{D_{2 h_{1}}}\right)^{2} \cdot t_{A_{4 h_{1}+2}} \cdots t_{A_{2 g}} t_{a_{g}} \\
& \sim\left(t_{A_{4 h_{1}+2}} \cdots t_{A_{2 g}} t_{a_{g}} t_{a_{g}} t_{A_{2 g}} \cdots t_{A_{4 h_{1}+2}} t_{D_{0}} t_{D_{1}} \cdots t_{D_{2 h_{1}}} t_{E_{h_{1}}}\right)^{2} .
\end{aligned}
$$

In order to prove this, we need a lemma.

Lemma 6.6. We deform $\Sigma_{g}^{2}$ as shown in Figure $8(a)$ and (b). Let $E$ and $E^{\prime}$ be the simple closed curves in $\Sigma_{g}^{2}$ as in Figure $8(a)$ and $(b)$, and let a be the arc connecting the boundary components of $\Sigma_{g}^{2}$ as in the figure. Then

$$
\begin{aligned}
t_{B_{0}} t_{B_{1}} \cdots t_{B_{g}}(E) & =E^{\prime}, \\
t_{B_{0}} t_{B_{1}} \cdots t_{B_{g}} t_{E}(a) & =t_{a_{g+1}} t_{a_{g+1}^{\prime}}(a) .
\end{aligned}
$$

Proof. From the equality (20), we see that

$$
t_{B_{0}} t_{B_{1}} \cdots t_{B_{g}}=\Delta_{2 g+1} \cdots \Delta_{2} \Delta_{1} t_{c_{r}}^{-1} .
$$

By drawing corresponding curves and applying the corresponding Dehn twist, we find that

$$
\Delta_{2 g+1} \cdots \Delta_{2} \Delta_{1} t_{c_{r}}^{-1}(E)=E^{\prime} \quad \text { and } \quad \Delta_{2 g+1} \cdots \Delta_{2} \Delta_{1} t_{c_{r}}^{-1} t_{E}(a)=t_{a_{g+1}} t_{a_{g+1}^{\prime}}(a) .
$$

This proves the lemma.
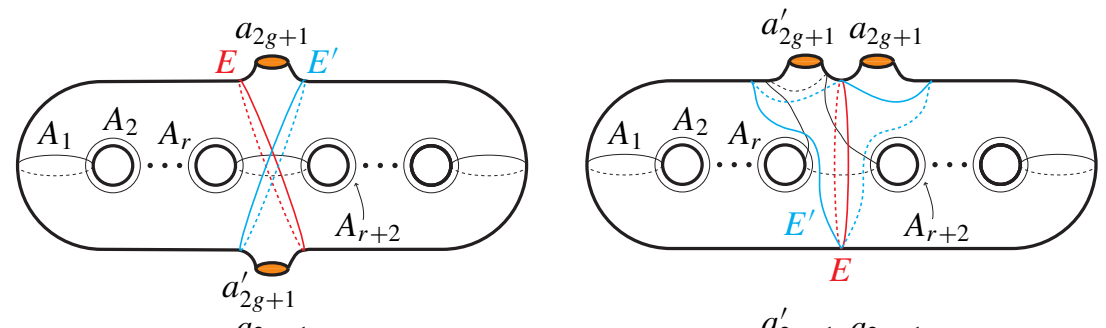

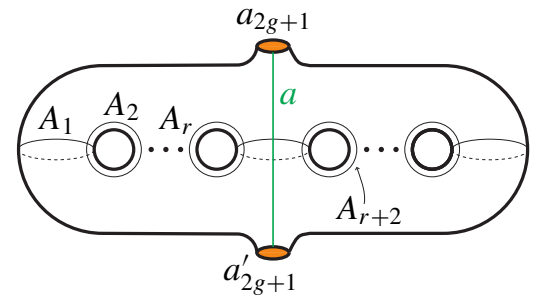

(a)

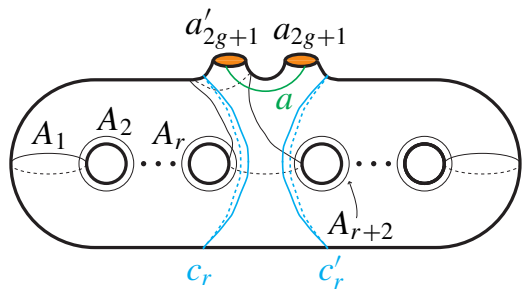

(b)

Figure 8. The curves $E, E^{\prime}$ and the $\operatorname{arc} a$. 
Proof of Proposition 6.5. For simplicity of notation, we write

$$
\tau:=t_{A_{4 h_{1}+2}} \cdots t_{A_{2 g}} t_{a_{g}} \quad \text { and } \quad \bar{\tau}:=t_{a_{g}} t_{A_{2 g}} \cdots t_{A_{4 h_{1}+2}} .
$$

Note that for each $i=2 h_{1}+2, \ldots, 2 g$, we find that

$$
t_{E_{h_{1}}} \tau \bar{\tau} t_{E_{h_{1}}}\left(A_{i}\right)=A_{i} \quad \text { and } \quad t_{E_{h_{1}}} \tau \bar{\tau} t_{E_{h_{1}}}\left(a_{g}\right)=a_{g} .
$$

This gives

$$
t_{E_{h_{1}}} \tau \bar{\tau} t_{E_{h_{1}}} \cdot t_{A_{i}} \sim t_{A_{i}} \cdot t_{E_{h_{1}}} \tau \bar{\tau} t_{E_{h_{1}}} \quad \text { and } t_{E_{h_{1}}} \tau \bar{\tau} t_{E_{h_{1}}} \cdot t_{a_{g}} \sim t_{a_{g}} \cdot t_{E_{h_{1}}} \tau \bar{\tau} t_{E_{h_{1}}} \text {, }
$$

so we obtain the relation

$$
t_{E_{h_{1}}} \tau \bar{\tau} t_{E_{h_{1}}} \cdot \tau \sim \tau \cdot t_{E_{h_{1}}} \tau \bar{\tau} t_{E_{h_{1}}} .
$$

Therefore, applying elementary transformations (including cyclic permutations) gives

$$
t_{E_{h_{1}}} \tau \bar{\tau} t_{E_{h_{1}}} \cdot \bar{\tau}\left(t_{D_{0}} t_{D_{1}} \cdots t_{D_{2 h_{1}}}\right)^{2} \cdot \tau \sim t_{E_{h_{1}}} \tau \bar{\tau} t_{E_{h_{1}}} \cdot \tau \cdot \bar{\tau}\left(t_{D_{0}} t_{D_{1}} \cdots t_{D_{2 h_{1}}}\right)^{2} .
$$

Since by drawing corresponding curves, applying the corresponding Dehn twist and (24) in Lemma 6.6, we have

$$
(\tau \bar{\tau})^{-1}\left(E_{h_{1}}\right)=t_{a_{2 h_{1}+1}} t_{a_{2 h_{1}+1}^{\prime}}^{\prime}\left(E_{h_{1}}\right)=t_{D_{0}} t_{D_{1}} \cdots t_{D_{2 h_{1}}}\left(E_{h_{1}}\right),
$$

we thus obtain

$$
\tau \bar{\tau} \cdot t_{D_{0}} t_{D_{1}} \cdots t_{D_{2 h_{1}}} \cdot t_{E_{h_{1}}} \sim t_{E_{h_{1}}} \cdot \tau \bar{\tau} \cdot t_{D_{0}} t_{D_{1}} \cdots t_{D_{2 h_{1}}} .
$$

Therefore, by using this relation, we have

$$
\begin{aligned}
t_{E_{h_{1}}} \tau \bar{\tau} t_{E_{h_{1}}} \cdot \tau \bar{\tau} \cdot\left(t_{D_{0}} t_{D_{1}} \cdots\right. & \left.t_{D_{2 h_{1}}}\right)^{2} \\
& \sim t_{E_{h_{1}}} \tau \bar{\tau} \cdot \tau \bar{\tau} \cdot t_{D_{0}} t_{D_{1}} \cdots t_{D_{2 h_{1}}} \cdot t_{E_{h_{1}}} \cdot t_{D_{0}} t_{D_{1}} \cdots t_{D_{2 h_{1}}} .
\end{aligned}
$$

By drawing corresponding curves, applying the corresponding Dehn twist and (23) in Lemma 6.6, we obtain

$$
(\tau \bar{\tau})^{-1}\left(A_{4 h_{1}+2}\right)=t_{D_{0}} t_{D_{1}} \cdots t_{D_{2 h_{1}}} t_{E_{h_{1}}}\left(A_{4 h_{1}+2}\right) .
$$

Therefore, we have

$$
\tau \bar{\tau} \cdot t_{D_{0}} t_{D_{1}} \cdots t_{D_{2 h_{1}}} t_{E_{h_{1}}} \cdot t_{A_{4 h_{1}+2}} \sim t_{A_{4 h_{1}+2}} \cdot \tau \bar{\tau} \cdot t_{D_{0}} t_{D_{1}} \cdots t_{D_{2 h_{1}}} t_{E_{h_{1}}} .
$$

Note that for each $i=4 h_{1}+3, \ldots, 2 g$, we find that

$$
\tau \bar{\tau}\left(A_{i}\right)=A_{i} \quad \text { and } \quad \tau \bar{\tau}\left(a_{g}\right)=a_{g} .
$$


Moreover, since $A_{4 h_{1}+3}, \ldots, A_{2 g}$ and $a_{g}$ are disjoint from $D_{0}, \ldots, D_{2 h_{1}}, E_{h_{1}}$, we therefore obtain, for each $i=2 h_{1}+3, \ldots, 2 g$,

$$
\begin{aligned}
& \tau \bar{\tau} t_{D_{0}} t_{D_{1}} \cdots t_{D_{2 h_{1}}} t_{E_{h_{1}}} \cdot t_{A_{i}} \sim t_{A_{i}} \cdot \tau \tau \bar{\tau} t_{D_{0}} t_{D_{1}} \cdots t_{D_{2 h_{1}}} t_{E_{h_{1}}}, \\
& \tau \bar{\tau} t_{D_{0}} t_{D_{1}} \cdots t_{D_{2 h_{1}}} t_{E_{h_{1}}} \cdot t_{a_{g}} \sim t_{a_{g}} \cdot \tau \bar{\tau} t_{D_{0}} t_{D_{1}} \cdots t_{D_{2 h_{1}}} t_{E_{h_{1}}} .
\end{aligned}
$$

This gives

$$
\tau \bar{\tau} \cdot \tau \bar{\tau} t_{D_{0}} t_{D_{1}} \cdots t_{D_{2 h_{1}}} t_{E_{h_{1}}} \sim \tau \bar{\tau} t_{D_{0}} t_{D_{1}} \cdots t_{D_{2 h_{1}}} t_{E_{h_{1}}} \cdot \tau \bar{\tau} .
$$

From this relation, applying elementary transformations (including cyclic permutations) gives

$$
\begin{aligned}
& t_{E_{h_{1}}} \tau \bar{\tau} \cdot \tau \bar{\tau} \cdot t_{D_{0}} t_{D_{1}} \cdots t_{D_{2 h_{1}}} t_{E_{h_{1}}} \cdot t_{D_{0}} t_{D_{1}} \cdots t_{D_{2 h_{1}}} \\
& \sim \tau \bar{\tau} \cdot t_{D_{0}} t_{D_{1}} \cdots t_{D_{2 h_{1}}} t_{E_{h_{1}}} \cdot \tau \bar{\tau} \cdot t_{D_{0}} t_{D_{1}} \cdots t_{D_{2 h_{1}}} \cdot t_{E_{h_{1}}} .
\end{aligned}
$$

Proposition 6.5 follows from the relations (25)-(27).

\section{Construction of simple loops $R_{1}, \ldots, R_{k}$}

In this section, we prove Proposition 5.2. This was based on Korkmaz's work [2009] and the argument in [Akhmedov and Ozbagci 2013]. In Proposition 4.3 of [Korkmaz 2009], he defined $l$ as $l=l\left(r_{1}\right)+\cdots+l\left(r_{k}\right)$. However, in this paper, it is sufficient to consider $l$ as $l=\max _{1 \leq i \leq k}\left\{l\left(r_{i}\right)\right\}$. Before providing the simple loops in $\Sigma_{g}$ in Proposition 5.2, we need the following proposition about simple loops $R_{1}, \ldots, R_{k}$ in $\Sigma_{n+l-1}$.

Proposition 7.1. Let $F_{n}$ be the subgroup of $\pi_{1}\left(\Sigma_{n}\right)$ generated by $a_{1}, \ldots, a_{n}$, i.e., $F_{n}$ is a free group of rank $n$. Let $r_{1}, \ldots, r_{k}$ be $k$ arbitrary elements in $F_{n}$ represented as words in $a_{1}, \ldots, a_{n}$. Let $l=\max _{1 \leq i \leq k}\left\{l\left(r_{i}\right)\right\}$, where $l\left(r_{i}\right)$ is the syllable length of $r_{i}$. Then there are simple loops $R_{1}, \ldots, R_{k}$ in $\Sigma_{n+l-1}$ with the property that for each $1 \leq i \leq k$ :

(a) $R_{i}$ is freely homotopic to a simple closed curve which intersects $a_{n+l-1}$ transversely at only one point.

(b) $\Phi\left(\left[R_{i}\right]\right)=r_{i}$, where $\left[R_{i}\right] \in \pi_{1}\left(\Sigma_{n+l-1}\right)$ is the homotopy class of $R_{i}$, and $\Phi: \pi_{1}\left(\Sigma_{n+l-1}\right) \rightarrow \pi_{1}\left(\Sigma_{n}\right)$ is the map defined by $\Phi\left(a_{j}\right)=a_{j}$ for $1 \leq j \leq n$ and $\Phi(\alpha)=1$ for $\alpha \in\left\{a_{n+1}, \ldots, a_{n+l-1}, b_{1}, \ldots, b_{n+l-1}\right\}$.

Proof. Let us consider the surface $\Sigma_{n}$ embedded in $\mathbb{R}^{3}$ as shown in Figure 1 such that for each $1 \leq j \leq n$, a simple closed curve $b_{j}^{\prime}$ in $\Sigma_{n}$ which is isotopic to $b_{j}$ lies on the plane $x=0$. Write $r_{i}=a_{i_{1}}^{m_{1}} \cdots a_{i_{d}}^{m_{d}}$, where $d=l\left(r_{i}\right)$ is the syllable length of $r_{i}$. We denote by $\xi$ a constant such that the base point lies in the plane $z=\xi$. Let $L$ be an arc in $\Sigma_{n}$ which lies in the half plane $\{z=\xi\} \cap\{x \geq 0\}$. 


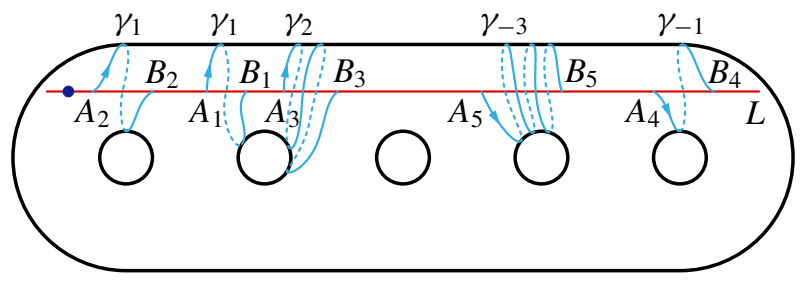

(a)

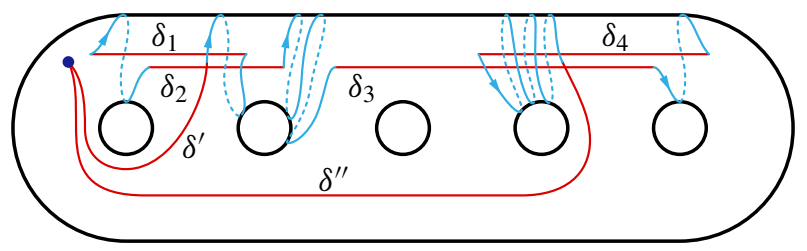

(b)

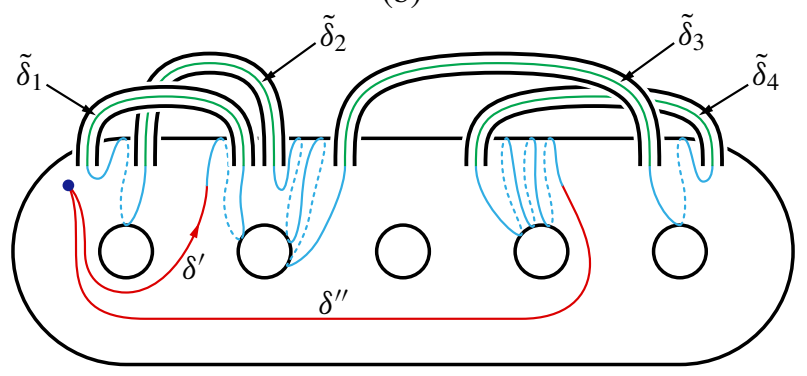

(c)

Figure 9. Construction of $R_{i}$ on $\Sigma_{n+d-1}$ for $r_{i}=a_{2} a_{1} a_{2}^{2} a_{5}^{-1} a_{4}^{-3}$ and for $n=5$.

For $1 \leq t \leq d$, let $\alpha_{t}$ be a loop in $\Sigma_{n}$ which is isotopic to $a_{i_{t}}$. If $j_{s}=j_{s^{\prime}}$ for some $s<s^{\prime}$, then we assume that $\alpha_{s^{\prime}}$ is to the right of $\alpha_{s}$ and that $\alpha_{s^{\prime}}$ is disjoint from $\alpha_{s}$. Here, right means the positive direction of the $y$-axis. Let $A_{t}$ (resp. $B_{t}$ ) be points on $L$ lying to the left (resp. right) of $\alpha_{t}$ such that there are no $A_{s}$ (resp. $B_{s}$ ) between $\alpha_{t}$ and $A_{t}$ (resp. $B_{t}$ ).

Let $\gamma_{m_{t}}=t_{\alpha_{t}}^{-m_{t}}\left(\zeta_{t}\right)$, where $\zeta_{t}$ is the subarc of $L$ from the point $A_{j}$ to the point $B_{j}$. For each $1 \leq j \leq d-1$, let $\delta_{j}$ denote the subarc of $L$ from the point $B_{j}$ to the point $A_{j+1}$. Then we can define an $\operatorname{arc} \beta$ in $\Sigma_{n}$ connecting $A_{1}$ to $B_{d}$ to be

$$
\beta=\gamma_{m_{1}} \star \delta_{1} \star \gamma_{m_{2}} \star \delta_{2} \star \cdots \star \delta_{d-1} \star \gamma_{m_{d}},
$$

where $\gamma \star \delta$ denotes an arc $\gamma$ followed by an arc $\delta$. Let $\delta_{0}$ be the subarc of $L$ from the base point to $A_{1}$, and $\delta_{d}$ the subarc from $B_{d}$ to the base point. Then $\delta_{0} \star \beta \star \delta_{d}$ represents $r_{i}$ (cf. Figure 9(a)). After perturbing $\beta$ slightly, we assume that $\delta_{1}, \ldots, \delta_{d-1}$ are pairwise disjoint and lie parallel to the plane $x=0$. Note that all self-intersection points of $\delta_{0} \star \beta \star \delta_{d}$ lie on $\delta_{0} \cup \delta_{1} \cup \cdots \cup \delta_{d}$. 
Let $\delta^{\prime}$ and $\delta^{\prime \prime}$ be arcs from the base point to $A_{1}$ and from $B_{d}$ to the base point, respectively, which are disjoint from $\alpha_{1}, \alpha_{2}, \ldots, \alpha_{d}$ and $b_{1}^{\prime}, b_{2}^{\prime}, \ldots, b_{n}^{\prime}$ and lie in the space $\{z \leq \xi\}$. Suppose that the interiors of $\delta^{\prime}, \delta^{\prime \prime}$ and $\beta$ are pairwise disjoint. Then the loop $\delta^{\prime} \star \beta \star \delta^{\prime \prime}$ represents

$$
b_{1} b_{2} \cdots b_{i_{1}-1} r_{i} b_{i_{d}}^{-1} \cdots b_{2}^{-1} b_{1}^{-1}
$$

in $\pi_{1}\left(\Sigma_{n}\right)$ (cf. Figure 9(b)).

Let $D_{1}, D_{1}^{\prime}, \ldots, D_{d-1}, D_{d-1}^{\prime}$ be pairwise disjoint disks in $\Sigma_{n}$ such that for each $1 \leq t \leq d-1, \operatorname{Int}\left(D_{t}\right)$ and $\operatorname{Int}\left(D_{t}^{\prime}\right)$ are disjoint from $\delta^{\prime}, \beta$ and $\delta^{\prime \prime}$, and $A_{t} \in \partial D_{t}$ and $B_{t} \in \partial D_{t}^{\prime}$. We remove $2 d-2$ open $\operatorname{disks} \operatorname{Int}\left(D_{t}\right)$ and $\operatorname{Int}\left(D_{t}^{\prime}\right)$ from $\Sigma_{n}$. Then for each $1 \leq t \leq d-1$, by attaching an annulus, denote by $\mathscr{A}_{t}$, to the surface

$$
\Sigma_{n} \backslash \bigcup_{t=1}^{d-1}\left(\operatorname{Int}\left(D_{t}\right) \cup \operatorname{Int}\left(D_{t}^{\prime}\right)\right)
$$

along $\partial D_{t}$ and $\partial D_{t}^{\prime}$, we obtain the closed oriented surface

$$
\left(\Sigma_{n} \backslash \bigcup_{t=1}^{d-1}\left(\operatorname{Int}\left(D_{t}\right) \cup \operatorname{Int}\left(D_{t}^{\prime}\right)\right)\right) \cap\left(\bigcup_{t=1}^{d-1} \mathscr{A}_{t}\right)
$$

of genus $n+d-1$, denoted by $\Sigma_{n+d-1}$. An orientation on $\Sigma_{n+d-1}$ is given by the orientation on $\Sigma_{n}$.

We define a loop $R_{i}$ in $\Sigma_{n+d-1}$ as follows. For each $1 \leq t \leq d-1$, let $\tilde{\delta}_{t}$ be a simple arc in $\mathscr{A}_{t}$ from the point $B_{t}$ to the point $A_{t+1}$ such that $\tilde{\delta}_{t}$ lies parallel to the plane $x=0$. Then by "replacing" $\delta_{t}$ in $\delta^{\prime} \star \beta \star \delta^{\prime \prime}$ by $\tilde{\delta}_{t}$, we obtain the loop

$$
R=\delta^{\prime} \star \gamma_{m_{1}} \star \tilde{\delta}_{1} \star \gamma_{m_{2}} \star \tilde{\delta}_{2} \star \cdots \star \tilde{\delta}_{d-1} \star \gamma_{m_{d}} \star \delta^{\prime \prime} .
$$

In particular, $R_{i}$ is simple in $\Sigma_{n+d-1}$ (cf. Figure 9(c)).

Note that from construction, $\tilde{\delta}_{t} \star \delta_{t}$ is a simple closed curve in $\Sigma_{n+d-1}$. If we collapse each $\mathscr{A}_{t}$ onto the arc $\delta_{t}$, then we obtain a map $\Sigma_{n+d-1} \rightarrow \Sigma_{n}$. The induced map $\pi_{1}\left(\Sigma_{n+d-1}\right) \rightarrow \pi_{1}\left(\Sigma_{n}\right)$ takes $[R]$ to

$$
b_{1} b_{2} \cdots b_{i_{1}-1} r_{i} b_{i_{d}}^{-1} \cdots b_{2}^{-1} b_{1}^{-1},
$$

which in turn is mapped to $r_{i}$ under the map $\pi_{1}\left(\Sigma_{n}\right) \rightarrow \pi_{1}\left(\Sigma_{n}\right)$ sending $a_{j}$ to $a_{j}$ and $b_{j}$ to 1 for all $j$.

Let $h=n+l-1$, where $l=\max _{1 \leq i \leq k}\left\{l\left(r_{i}\right)\right\}$. For each $1 \leq i \leq k$, we now construct a loop $R_{i}$ in $\Sigma_{h}$ as follows. First, by sliding $\mathscr{A}_{1}, \ldots, \mathscr{A}_{l\left(r_{i}\right)-1}$, we deform the surface $\Sigma_{n+l\left(r_{i}\right)-1}$ into the standard position as shown in Figure 1 in such a way that the simple loop $\tilde{\delta}_{t} \star \delta_{t}$ becomes isotopic to $b_{n+t}$ and the boundary curves of $A_{t}$ become isotopic to $a_{n+t}$ (cf. Figure 10(a), (b) and (c)). If $l\left(r_{j}\right)=l$ for some $j$, then we see that the simple closed curve $a_{h}$ intersects $R_{j}$ transversely at one point. 


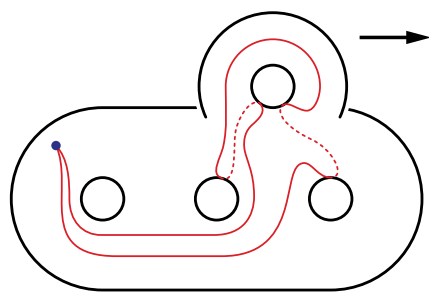

(a)

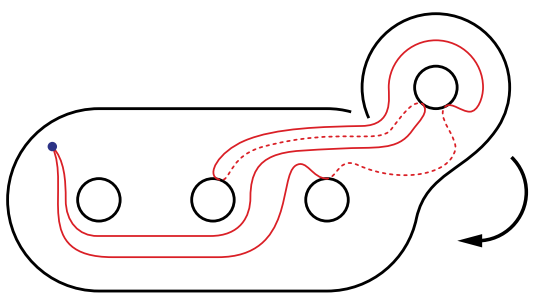

(b)

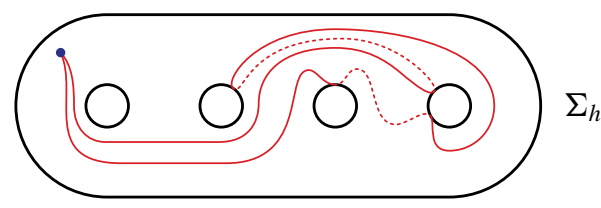

(c)

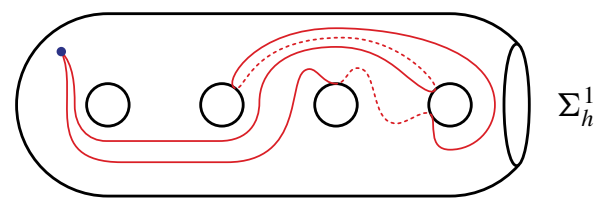

(d)

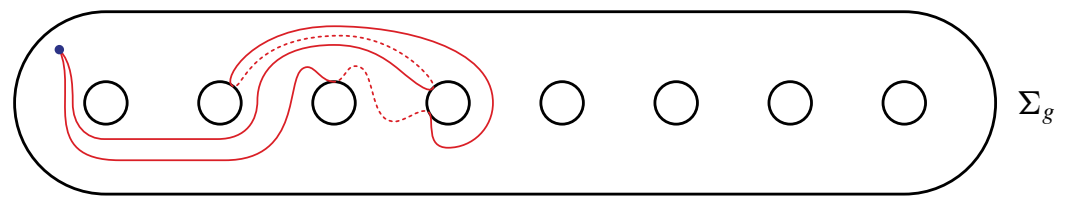

(e)

Figure 10. Construction of $R_{i}$ for $r_{i}=a_{3}^{-1} a_{2}^{-1}$ in the case $n=3$ and $g=8$.

Therefore, we assume that $l\left(r_{i}\right)<l$. Next, we remove a small open disk from the deformed surface near $a_{n+l\left(r_{i}\right)-1}$ and disjoint from $R_{i}$ (cf. Figure 10(d)). Thus, we obtain a surface of genus $n+l\left(r_{i}\right)-1$ with one boundary component, denoted by $\Sigma_{n+l\left(r_{i}\right)-1}^{1}$. We embed $\Sigma_{n+l\left(r_{i}\right)-1}^{1}$ into the standard surface $\Sigma_{h}$ in such a way that for each $1 \leq t \leq n+l\left(r_{i}\right)-1$, simple loops $a_{t}, b_{t}$ in $\Sigma_{n+l\left(r_{i}\right)-1}^{1}$ correspond to the simple loops $a_{t}, b_{t}$ in $\Sigma_{h}$ (cf. Figure 10(e)). Finally, we replace $R_{i}$ with a simple representative of $\left[R_{i}\right]\left(\left(b_{1} b_{2} \cdots b_{h-1}\right)\left(b_{1} b_{2} \cdots b_{h}\right)^{-1}\right)^{\epsilon}$, where $\epsilon= \pm 1$ (cf. Figure 10(d)). Then we see that the resulting simple loop $R_{i}$ intersects $a_{h}$ transversely at one point.

From the above construction, $\Phi: \pi_{1}\left(\Sigma_{h}\right) \rightarrow \pi_{1}\left(\Sigma_{n}\right)$ maps $\left[R_{i}\right]$ to $r_{i}$ for each $i=1, \ldots, k$. This gives the required simple loops $R_{1}, \ldots, R_{k}$.

Proof of Proposition 5.2. Consider a surface $\Sigma_{n+l-1}$ and the loops $R_{1}, \ldots, R_{k}$ constructed in Proposition 7.1. We remove a small open disk from $\Sigma_{n+l-1}$ near $a_{n+l-1}$ and disjoint from all $R_{i}$ (cf. Figure 11(a)). Denote by $\Sigma_{n+l-1}^{1}$ the resulting surface of genus $n+l-1$ with one boundary component. We embed $\Sigma_{n+l-1}^{1}$ into the standard surface $\Sigma_{g}$ in such a way that for each $1 \leq t \leq n+l-1$, simple loops $a_{t}, b_{t}$ in $\Sigma_{n+l-1}^{1}$ correspond to the simple loops $a_{t}, b_{t}$ in $\Sigma_{g}$ (cf. Figure 11(b)). Then we can modify $R_{1}, \ldots, R_{k}$ so that each $R_{i}(i=1, \ldots, k)$ satisfies the property of Proposition 5.2 


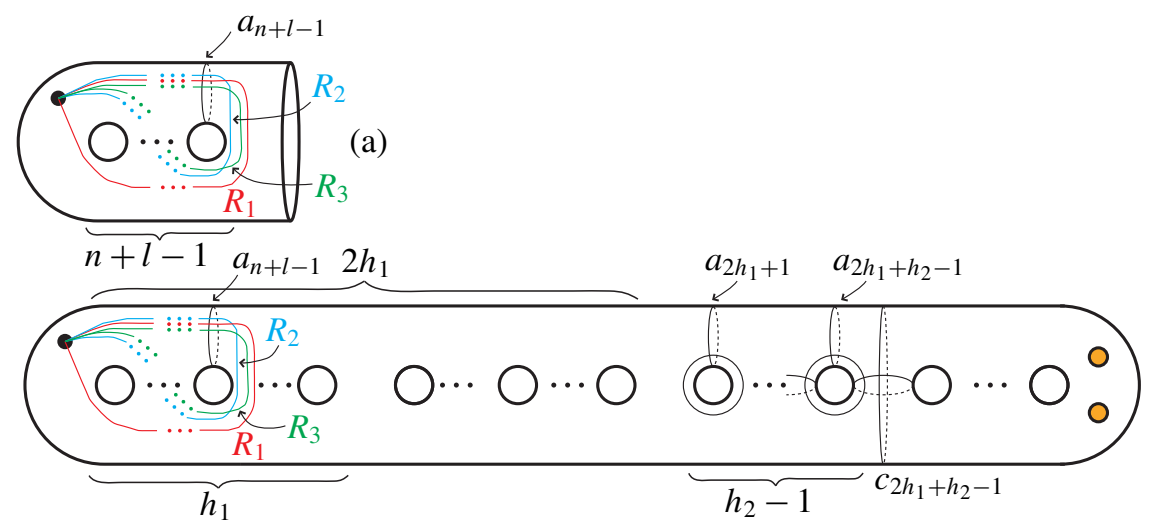

Figure 11. Modified curves $R_{1}, \ldots, R_{k}$ in $\Sigma_{g}$.

by replacing $R_{i}$ with a simple representative of $\left[R_{i}\right]\left(b_{2 h_{1}+1} b_{2 h_{2}+2} \cdots b_{2 h_{1}+h_{2}-i}\right)^{\epsilon}$ if $i$ is odd, and $\left[R_{i}\right] a_{2 h_{1}+h_{2}-i}^{\epsilon}$ if $i$ is even, where $\epsilon= \pm 1$ (cf. Figure 5). Therefore, we obtain the required simple loops $R_{1}, \ldots, R_{k}$.

\section{Remarks}

The results of [Gompf 1995; Donaldson 1999; Gompf and Stipsicz 1999] mentioned in the introduction naturally raise the following two basic questions, which remain open.

Question 8.1 (cf. [Korkmaz and Stipsicz 2009]). Given a symplectic 4-manifold, what is the minimal genus $g$ for which it has a genus- $g$ Lefschetz pencil?

Question 8.2. Given a finitely presented group $\Gamma$, what is the minimal genus, denoted by $g_{P}(\Gamma)$, for which there exists a genus- $g$ Lefschetz pencil on a symplectic 4-manifold with fundamental group $\Gamma$ ?

Although these two questions remain open, for Question 8.2, we can give an upper bound for $g_{P}(\Gamma)$ as a corollary of Theorem 1.2.

Corollary 8.3. We have $g_{P}(\Gamma) \leq 4(n+l-1)+k$ for $k \geq 1$, and $g_{P}\left(F_{n}\right) \leq 4 n+2$.

However, this upper bound for $g_{P}(\Gamma)$ may not be sharp. In fact, since $\mathbb{C P}^{2}$ admits a genus- 0 Lefschetz pencil, $g_{P}(\Gamma)=0$ if $\Gamma$ is the trivial group. When we replace the relations in Proposition 5.1 and the map $\psi_{k}$ in Section 5A by another relation and map, we can improve the upper bound of $g_{P}(\Gamma)$. For example, for every positive integer $n$, the article [Hamada et al. $\geq 2016$ ] gave a genus- $g$ Lefschetz pencil on a 4-manifold $X_{n}$ such that $\pi_{1}\left(X_{n}\right) \cong \mathbb{Z} \oplus \mathbb{Z}_{n}$ for every $g \geq 4$ using a similar construction to this paper. Therefore, $g_{P}\left(\mathbb{Z} \oplus \mathbb{Z}_{m}\right) \leq 4$. 
We expect that by a combination of substitution techniques and partial conjugation techniques, we could obtain results for Lefschetz fibrations with (-1)-sections analogous to those obtained by fiber sum operations. The articles [Ozbagci and Stipsicz 2000; Korkmaz 2001; Monden 2014] gave examples of nonholomorphic Lefschetz fibrations by fiber sum operations (and lantern substitutions). By a similar technique to this paper (and a lantern substitution), two kinds of nonholomorphic Lefschetz fibrations with $(-1)$-sections were constructed in [Hamada et al. $\geq 2016$ ]. One is a Lefschetz fibration with noncomplex total space, and the other is a Lefschetz fibration violating the "slope inequality".

Finally, we explain why the Lefschetz fibrations constructed in [Korkmaz 2009; Akhmedov and Ozbagci 2013; Kobayashi 2015] do not have (-1)-sections. In [Korkmaz 2009; Kobayashi 2015], twisted fiber sum operations were adopted, and the fibrations in [Akhmedov and Ozbagci 2013] were obtained by performing Luttinger surgeries and knot surgeries on the symplectic sum of certain symplectic 4-manifolds. The fiber sum of Lefschetz fibrations has no (-1)-sections (see [Stipsicz 2001a], and also [Smith 2001]). In particular, the symplectic sum of symplectic 4-manifolds is minimal, that is, it does not contain any $(-1)$-spheres (see [Usher 2006], and also [Sato 2006; Baykur 2015]), and Luttinger surgery and knot surgery preserve minimality of symplectic 4-manifolds from the result of [Usher 2006]. Therefore, we see that the fibrations in [Korkmaz 2009; Akhmedov and Ozbagci 2013; Kobayashi 2015] do not have any (-1)-sections.

\section{Acknowledgments}

The authors would like to thank Susumu Hirose for his comments on this paper. The second author was supported by Grant-in-Aid for Young Scientists (B) (No. 13276356), Japan Society for the Promotion of Science.

\section{References}

[Akbulut and Ozbagci 2002] S. Akbulut and B. Ozbagci, "On the topology of compact Stein surfaces", Int. Math. Res. Not. 2002:15 (2002), 769-782. MR 2003a:57049 Zbl 1007.57023

[Akhmedov and Ozbagci 2013] A. Akhmedov and B. Ozbagci, "Exotic Stein fillings with arbitrary fundamental group", preprint, 2013. arXiv 1212.1743

[Amorós et al. 2000] J. Amorós, F. Bogomolov, L. Katzarkov, and T. Pantev, "Symplectic Lefschetz fibrations with arbitrary fundamental groups", J. Differential Geom. 54:3 (2000), 489-545. MR 2002g:57051 Zbl 1031.57021

[Auroux 2006a] D. Auroux, “The canonical pencils on Horikawa surfaces”, Geom. Topol. 10 (2006), 2173-2217. MR 2007m:14065 Zbl 1129.57030

[Auroux 2006b] D. Auroux, "Mapping class group factorizations and symplectic 4-manifolds: some open problems", pp. 123-132 in Problems on mapping class groups and related topics, edited by B. Farb, Proceedings of Symposia in Pure Mathematics 74, American Mathematical Society, Providence, RI, 2006. MR 2007h:53134 Zbl 1304.57027 
[Baykur 2015] R. I. Baykur, "Minimality and fiber sum decompositions of Lefschetz fibrations", Proc. Amer. Math. Soc. (online publication December 2015).

[Cadavid 1998] C. A. Cadavid, On a remarkable set of words in the mapping class group, thesis, University of Texas, Austin, TX, 1998, Available at http://search.proquest.com/docview/304458143. MR 2699379

[Donaldson 1999] S. K. Donaldson, "Lefschetz pencils on symplectic manifolds", J. Differential Geom. 53:2 (1999), 205-236. MR 2002g:53154 Zbl 1040.53094

[Etnyre and Honda 2002] J. B. Etnyre and K. Honda, "On symplectic cobordisms", Math. Ann. 323:1 (2002), 31-39. MR 2003c:57026 Zbl 1022.53059

[Gompf 1995] R. E. Gompf, "A new construction of symplectic manifolds", Ann. of Math. (2) 142:3 (1995), 527-595. MR 96j:57025 Zbl 0849.53027

[Gompf and Stipsicz 1999] R. E. Gompf and A. I. Stipsicz, 4-manifolds and Kirby calculus, Graduate Studies in Mathematics 20, American Mathematical Society, Providence, RI, 1999. MR 2000h:57038 Zbl 0933.57020

[Gurtas 2004] Y. Z. Gurtas, "Positive Dehn twist expressions for some new involutions in mapping class group", preprint, 2004. arXiv math/0404310

[Hamada $\geq 2016$ ] N. Hamada, "On a combinatorial decomposition for relations among Dehn twists", In preparation.

[Hamada et al. $\geq 2016]$ M. Hamada, R. Kobayashi, and N. Monden, "Non-holomorphic Lefschetz fibrations with (-1)-sections", In preparation.

[Kas 1980] A. Kas, "On the handlebody decomposition associated to a Lefschetz fibration", Pacific J. Math. 89:1 (1980), 89-104. MR 82f:57012 Zbl 0457.14011

[Kobayashi 2015] R. Kobayashi, "On genera of Lefschetz fibrations and finitely presented groups", Osaka J. Math. (online publication January 2015).

[Korkmaz 2001] M. Korkmaz, "Noncomplex smooth 4-manifolds with Lefschetz fibrations”, Int. Math. Res. Not. 2001:3 (2001), 115-128. MR 2001m:57036 Zbl 0977.57020

[Korkmaz 2009] M. Korkmaz, "Lefschetz fibrations and an invariant of finitely presented groups", Int. Math. Res. Not. 2009:9 (2009), 1547-1572. MR 2010c:57037 Zbl 1173.57013

[Korkmaz and Ozbagci 2008] M. Korkmaz and B. Ozbagci, "On sections of elliptic fibrations", Michigan Math. J. 56:1 (2008), 77-87. MR 2009f:57043 Zbl 1158.57033

[Korkmaz and Stipsicz 2009] M. Korkmaz and A. I. Stipsicz, "Lefschetz fibrations on 4-manifolds", pp. 271-296 in Handbook of Teichmüller theory, vol. 2, edited by A. Papadopoulos, IRMA Lectures in Mathematics and Theoretical Physics 13, European Mathematical Society, Zürich, 2009. MR 2010k:57048 Zbl 1177.57001

[Loi and Piergallini 2001] A. Loi and R. Piergallini, "Compact Stein surfaces with boundary as branched covers of $B^{4 ",}$ Invent. Math. 143:2 (2001), 325-348. MR 2002c:53139 Zbl 0983.32027

[Matsumoto 1996] Y. Matsumoto, "Lefschetz fibrations of genus two: a topological approach", pp. 123-148 in Topology and Teichmüller spaces (Katinkulta, 1995), edited by S. Kojima et al., World Scientific, River Edge, NJ, 1996. MR 2000h:14038 Zbl 0921.57006

[Monden 2014] N. Monden, "Lefschetz fibrations with small slope", Pacific J. Math. 267:1 (2014), 243-256. MR 3163482 Zbl 1306.57019

[Ozbagci and Stipsicz 2000] B. Ozbagci and A. I. Stipsicz, "Noncomplex smooth 4-manifolds with genus-2 Lefschetz fibrations”, Proc. Amer. Math. Soc. 128:10 (2000), 3125-3128. MR 2000m:57036 Zbl 0951.57015

[Sato 2006] Y. Sato, “The Stipsicz's conjecture for genus-2 Lefschetz fibrations”, preprint, 2006. 
[Smith 2001] I. Smith, "Geometric monodromy and the hyperbolic disc", Q. J. Math. 52:2 (2001), 217-228. MR 2002c:57046 Zbl 0981.57013

[Stipsicz 2001a] A. I. Stipsicz, "Indecomposability of certain Lefschetz fibrations", Proc. Amer. Math. Soc. 129:5 (2001), 1499-1502. MR 2001h:57029 Zbl 0978.57022

[Stipsicz 2001b] A. I. Stipsicz, "Spin structures on Lefschetz fibrations", Bull. London Math. Soc. 33:4 (2001), 466-472. MR 2002a:53062 Zbl 1037.57019

[Tanaka 2012] S. Tanaka, "On sections of hyperelliptic Lefschetz fibrations", Algebr. Geom. Topol. 12:4 (2012), 2259-2286. MR 3020206 Zbl 1268.57010

[Usher 2006] M. Usher, "Minimality and symplectic sums", Int. Math. Res. Not. 2006 (2006), Art. ID \#49857. MR 2007h:53139 Zbl 1110.57017

Received March 27, 2015. Revised September 22, 2015.

RYOMA KOBAYASHI

DEPARTMENT OF GENERAL EDUCATION

ISHIKAWA NATIONAL COLLEGE OF TECHNOLOGY

TSUBATA, ISHIKAWA 929-0392

JAPAN

kobayashi_ryoma@ishikawa-nct.ac.jp

NAOYUKI MONDEN

DEPARTMENT OF ENGINEERING SCIENCE

OSAKA ELECTRO-COMMUNICATION UNIVERSITY

HATSU-CHO 18-8

NEYAGAWA 572-8530

JAPAN

monden@isc.osakac.ac.jp 


\title{
PACIFIC JOURNAL OF MATHEMATICS
}

\author{
msp.org/pjm
}

Founded in 1951 by E. F. Beckenbach (1906-1982) and F. Wolf (1904-1989)

\section{EDITORS}

Don Blasius (Managing Editor)

Department of Mathematics

University of California

Los Angeles, CA 90095-1555

blasius@math.ucla.edu

\author{
Paul Balmer \\ Department of Mathematics \\ University of California \\ Los Angeles, CA 90095-1555 \\ balmer@math.ucla.edu \\ Robert Finn \\ Department of Mathematics \\ Stanford University \\ Stanford, CA 94305-2125 \\ finn@math.stanford.edu \\ Sorin Popa \\ Department of Mathematics \\ University of California \\ Los Angeles, CA 90095-1555 \\ popa@math.ucla.edu
}

\author{
Vyjayanthi Chari \\ Department of Mathematics \\ University of California \\ Riverside, CA 92521-0135 \\ chari@math.ucr.edu \\ Kefeng Liu \\ Department of Mathematics \\ University of California \\ Los Angeles, CA 90095-1555 \\ liu@math.ucla.edu \\ Jie Qing \\ Department of Mathematics \\ University of California \\ Santa Cruz, CA 95064 \\ qing@ cats.ucsc.edu
}

\section{PRODUCTION}

Silvio Levy, Scientific Editor, production@msp.org

\section{SUPPORTING INSTITUTIONS}

ACADEMIA SINICA, TAIPEI

CALIFORNIA INST. OF TECHNOLOGY

INST. DE MATEMÁTICA PURA E APLICADA

KEIO UNIVERSITY

MATH. SCIENCES RESEARCH INSTITUTE

NEW MEXICO STATE UNIV.

OREGON STATE UNIV.

\author{
STANFORD UNIVERSITY \\ UNIV. OF BRITISH COLUMBIA \\ UNIV. OF CALIFORNIA, BERKELEY \\ UNIV. OF CALIFORNIA, DAVIS \\ UNIV. OF CALIFORNIA, LOS ANGELES \\ UNIV. OF CALIFORNIA, RIVERSIDE \\ UNIV. OF CALIFORNIA, SAN DIEGO \\ UNIV. OF CALIF., SANTA BARBARA
}

\author{
Daryl Cooper \\ Department of Mathematics \\ University of California \\ Santa Barbara, CA 93106-3080 \\ cooper@math.ucsb.edu \\ Jiang-Hua Lu \\ Department of Mathematics \\ The University of Hong Kong \\ Pokfulam Rd., Hong Kong \\ jhlu@maths.hku.hk \\ Paul Yang \\ Department of Mathematics \\ Princeton University \\ Princeton NJ 08544-1000 \\ yang@math.princeton.edu
}

These supporting institutions contribute to the cost of publication of this Journal, but they are not owners or publishers and have no responsibility for its contents or policies.

See inside back cover or msp.org/pjm for submission instructions.

The subscription price for 2016 is US $\$ 440 /$ year for the electronic version, and $\$ 600 /$ year for print and electronic.

Subscriptions, requests for back issues and changes of subscriber address should be sent to Pacific Journal of Mathematics, P.O. Box 4163, Berkeley, CA 94704-0163, U.S.A. The Pacific Journal of Mathematics is indexed by Mathematical Reviews, Zentralblatt MATH, PASCAL CNRS Index, Referativnyi Zhurnal, Current Mathematical Publications and Web of Knowledge (Science Citation Index).

The Pacific Journal of Mathematics (ISSN 0030-8730) at the University of California, c/o Department of Mathematics, 798 Evans Hall \#3840, Berkeley, CA 94720-3840, is published twelve times a year. Periodical rate postage paid at Berkeley, CA 94704, and additional mailing offices. POSTMASTER: send address changes to Pacific Journal of Mathematics, P.O. Box 4163, Berkeley, CA 94704-0163.

PJM peer review and production are managed by EditFLOW ${ }^{\circledR}$ from Mathematical Sciences Publishers.

\section{PUBLISHED BY}

\section{mathematical sciences publishers \\ nonprofit scientific publishing}

http://msp.org/

(C) 2016 Mathematical Sciences Publishers 


\section{PACIFIC JOURNAL OF MATHEMATICS}

Volume $282 \quad$ No. $2 \quad$ June 2016

Exhausting curve complexes by finite rigid sets

JAVIER ARAMAYONA and CHRISTOPHER J. LEININGER

A variational characterization of flat spaces in dimension three

Giovanni Catino, Paolo Mastrolia and Dario D. Monticelli

Estimates of the gaps between consecutive eigenvalues of Laplacian

293

DAgUang Chen, TAO Zheng and HongCANG YANG

Liouville type theorems for the $p$-harmonic functions on certain manifolds

JINGYI CHEN and YUE WANG

Cartan-Fubini type rigidity of double covering morphisms of quadratic manifolds

\section{HOSUNG KIM}

On the uniform squeezing property of bounded convex domains in $\mathbb{C}^{n}$

KANG-TAE KIM and LIYOU ZHANG

Lefschetz pencils and finitely presented groups

RYOMA KOBAYASHI and NAOYUKI MONDEN

Knot homotopy in subspaces of the 3-sphere

YUYA KODA and MAKOTO OzAWA

On the relationship of continuity and boundary regularity in prescribed mean curvature Dirichlet problems

KIRK E. LANCASTER and JARON MELIN

Bridge spheres for the unknot are topologically minimal

JUNG HOON LEE

On the geometric construction of cohomology classes for cocompact discrete subgroups of $\mathrm{SL}_{n}(\mathbb{R})$ and $\mathrm{SL}_{n}(\mathbb{C})$

SUSANNE SCHIMPF

On Blaschke's conjecture

Xiaole Su, Hongwei Sun and Yusheng Wang

The role of the Jacobi identity in solving the Maurer-Cartan structure equation 